\title{
Relationships between HI Gas Mass, Stellar Mass, and the Star Formation Rate of HICAT+WISE (H I-WISE) Galaxies
}

\author{
Vaishali Parkash ${ }^{1}$ (D), Michael J. I. Brown ${ }^{1}$ (D), T. H. Jarrett ${ }^{2}$ (iD), and Nicolas J. Bonne ${ }^{3}$ (1) \\ ${ }^{1}$ School of Physics and Astronomy, Monash Centre for Astrophysics (MoCA), Monash University, Clayton, Victoria 3800, Australia; vaishali.parkash@ monash.edu \\ ${ }^{2}$ Astrophysics, Cosmology and Gravity Centre (ACGC), Astronomy Department, University of Cape Town, Private Bag X3, Rondebosch 7701, South Africa \\ ${ }^{3}$ Institute for Cosmology and Gravitation, Dennis Sciama Building, University of Portsmouth, Burnaby Road, Portsmouth PO1 3FX, UK \\ Received 2017 October 1; revised 2018 June 3; accepted 2018 June 29; published 2018 August 29
}

\begin{abstract}
We have measured the relationships between H I mass, stellar mass, and star formation rate using the HI Parkes All-Sky Survey Catalog (HICAT) and the Wide-field Infrared Survey Explorer (WISE). Of the 3513 HICAT sources, we find $3.4 \mu \mathrm{m}$ counterparts for 2896 sources (80\%), and provide new WISE-matched aperture photometry for these galaxies. For our principal sample of spiral galaxies with $W 1 \leqslant 10$ mag and $z \leqslant 0.01$, we identify H I detections for $93 \%$ of the sample. We measure lower $\mathrm{H} \mathrm{I}$-stellar mass relationships for $\mathrm{H}$ I-selected samples that do not include spiral galaxies with little H I gas. Our observations of the spiral sample show that H I mass increases with stellar mass with a power-law index of 0.35 ; however, this value is dependent on T-type, which affects both the median and the dispersion of HI mass. We also observe an upper limit on the H I gas fraction, which is consistent with a halo spin parameter model. We measure the star formation efficiency of spiral galaxies to be constant at $10^{-9.57} \mathrm{yr}^{-1} \pm 0.4$ dex for 2.5 orders of magnitude in stellar mass, despite the higher stellar mass spiral showing evidence of quenched star formation.
\end{abstract}

Key words: galaxies: evolution - galaxies: fundamental parameters - galaxies: spiral - galaxies: star formation radio lines: galaxies - radio lines: ISM

Supporting material: machine-readable table

\section{Introduction}

Star formation is fueled by atomic (H I) and molecular hydrogen, so we expect correlations between H I mass, stellar mass, and star formation rates (SFRs). This is exemplified by the Kennicutt-Schmidt law, which establishes a correlation between gas surface density and SFR, albeit both for H I and molecular gas, and with considerable scatter (Kennicutt 1998). Correlations are also observed between stellar mass and $\mathrm{HI}$ mass (e.g., Catinella et al. 2010; Huang et al. 2012; Maddox et al. 2015), although these are partially explained by luminosity-luminosity correlations. However, the presence of mass quenching indicates that $\mathrm{HI}$ mass declines above some high stellar mass threshold (e.g., Kauffmann et al. 2003; Gabor et al. 2010).

Although the relationship between H I mass and SFR in a galaxy is predicted by the Kennicutt-Schmidt law, there is a scatter of $\sim 0.2$ dex (Kennicutt 1998), implying that H I mass is just one of several factors that regulate SFR. Multiple studies using different surveys find that $\mathrm{HI}$ mass $\left(M_{\mathrm{H}}\right)$ increases with SFR (Mirabel \& Sanders 1988; Doyle \& Drinkwater 2006). A $\mathrm{H}$ I disk is a precondition for star formation, but star formation also requires gas accretion from the intergalactic medium to drive the $\mathrm{H}$ I gas inward, thereby cooling and converting it into molecular hydrogen (e.g., Prochaska \& Wolfe 2009; Obreschkow et al. 2016). By comparison, the relationship between molecular hydrogen and SFR is better understood since stars form in molecular clouds. Recent works (Leroy et al. 2008; Schruba et al. 2011) have shown that molecular hydrogen, rather than $\mathrm{HI}$, drives the Kennicutt-Schmidt law, but an empirical study on the relationship between molecular hydrogen and SFR using a large sample of galaxies is difficult to perform because of the lack of large-scale CO surveys. Also, there is uncertainty in converting $\mathrm{CO}$ intensity to molecular hydrogen content due to the uncertainty in the X-factor (Bolatto et al. 2013). Although H I gas is only indirectly related to the SFR, the results of measurements of H I gas can be used to imply information about the molecular hydrogen of a galaxy (e.g., Leroy et al. 2008; Wong et al. 2016).

Multiple studies have found that $\mathrm{HI}$ mass increases with stellar mass (Catinella et al. 2010; Huang et al. 2012; Maddox et al. 2015), which is partially explained by luminosityluminosity correlations. However, the relations measured by different studies disagree with each other. Huang et al. (2012) found $\left\langle\log M_{\mathrm{H}_{\mathrm{I}}}\right\rangle \propto 0.712\left\langle\log M_{*}\right\rangle$ for $M_{*} \leqslant 10^{9} M_{\odot}$ and $\left\langle\log M_{\mathrm{H}}\right\rangle \propto 0.276\left\langle\log M_{*}\right\rangle$ for $M_{*}>10^{9} M_{\odot}$. The break in the relationship represents the transition from irregular, low stellar mass galaxies to high mass, disk galaxies (Kereš et al. 2009; Maddox et al. 2015). However, Catinella et al. (2010) observed that $\mathrm{HI}$ mass increases only slightly with stellar mass (to the power of 0.02) for galaxies with $M_{*}>10^{9} M_{\odot}$.

The differences in the relation between $\mathrm{H}$ I mass and stellar mass in the literature may be due to sample selection. The samples of Huang et al. (2012) and Maddox et al. (2015) use the HI Arecibo Legacy Fast ALFA (ALFALFA) survey population, while the GASS sample of Catinella et al. (2010) is selected by stellar mass $\left(M_{*}>10^{10} M_{\odot}\right)$. By definition, the H I-selected samples of Huang et al. (2012) and Maddox et al. (2015) are more H I-rich compared to stellar mass-selected samples, and are biased against high stellar mass elliptical galaxies with very little $\mathrm{H}$ I gas, resulting in elevated trends for H I mass versus stellar masses. While the literature shows that H I mass increases with stellar mass, perhaps with a break or a plateau at high stellar masses (i.e., Catinella et al. 2010; Huang et al. 2012; Maddox et al. 2015), there are quantitative disagreements that we suspect are the result of sample selections. 
How the H I-based star formation efficiency (SFE), or its inverse, the HI-depletion timescale $\left(t_{\mathrm{dep}}\right)$, varies with a galaxy's stellar mass is unclear from the current literature. Huang et al. (2012), Jaskot et al. (2015), and Lutz et al. (2017) found that SFE increases with stellar mass for $\mathrm{HI}$-selected samples. Therefore, low mass galaxies that are more H I-rich than high stellar mass galaxies are inefficient at using their fuel reservoirs to form stars. Contradicting this, Schiminovich et al. (2010) found the SFE of massive galaxies $\left(M_{*}>10^{10} M_{\odot}\right)$ to be constant at $10^{-9.5} \mathrm{yr}^{-1}$, and Wong et al. (2016) found SFE to be constant at $10^{-9.65} \mathrm{yr}^{-1}$ across five orders of magnitude of stellar mass for star-forming galaxies. Incompleteness and sample size are issues for relations from the prior literature, with Jaskot et al. (2015) having Wide-field Infrared Survey Explorer (WISE) detections for $63 \%$ of their H I sources, while Wong et al. (2016) is highly complete, containing just 84 galaxies.

A key limitation of previous studies is low completeness, particularly for infrared counterparts for H I sources, which facilitate the measurement of stellar masses and SFRs. Doyle \& Drinkwater (2006) used fluxes from the Infrared Astronomical Satellite (IRAS) to calculate SFRs for galaxies in the HI Parkes All-Sky Survey (HIPASS) optical catalog (HOPCAT) (Doyle et al. (2005). Due to the 0.5 angular resolution and $0.7 \mathrm{Jy} 10 \sigma$ sensitivity of the IRAS at $12 \mu \mathrm{m}$, they only found infrared counterparts for $32 \%$ of the HIPASS catalog (HICAT). Their final sample comprised galaxies with high SFRs and excluded galaxies with low rates of star formation, including elliptical galaxies and dwarf galaxies, because at $z=0.01$ the IRAS can only detect sources brighter than $\nu L_{\nu} \sim 1 \times 10^{10} L_{\odot}$. Jaskot et al. (2015) improved previous studies by using WISE, which has a $12 \mu \mathrm{m} 5 \sigma$ sensitivity of $1 \mathrm{mJy}$, but at the $z \sim 0.06$ maximum redshift of ALFALFA galaxies WISE detects galaxies brighter than $\nu L_{\nu} \sim 6 \times 10^{8} L_{\odot}$, which resulted in Jaskot et al. (2015) finding infrared counterparts for just $63 \%$ of their H I sources. To improve on the prior literature, we need large samples of galaxies that are highly complete for H I counterparts, while probing large ranges of stellar mass, H I mass, and SFR.

For this work, we measure the relationship between $\mathrm{HI}$ mass, stellar mass, and SFR using three galaxy samples combining HICAT and WISE. The paper is arranged as follows: Section 2 describes the data used in this research; Section 3 details the equations used to calculate the masses and SFRs; Section 4 describes the samples; Sections 5 and 6 present the results; Section 7 discusses the results; and Section 8 summarizes our work. All magnitudes are in the Vega system. The cosmology applied in this paper is $H_{0}=$ $70 \mathrm{~km} \mathrm{~s}^{-1}, \Omega_{M}=0.3$, and $\Omega_{\Lambda}=0.7$.

\section{Data}

\subsection{HICAT}

The principal source of data for our analysis is HICAT, which is derived from HIPASS (Barnes et al. 2001; Meyer et al. 2004). HIPASS is a blind survey below a declination $\delta$ of $+2^{\circ}$, performed with the Parkes $64 \mathrm{~m}$ radio telescope using a $21 \mathrm{~cm}$ multibeam receiver. HICAT contains $4315 \mathrm{HI}$ sources selected from HIPASS and is $99 \%$ complete at a peak flux of $84 \mathrm{mJy}$ and an integrated flux of $9.4 \mathrm{Jy} \mathrm{km} \mathrm{s}^{-1}$ (Meyer et al. 2004; Zwaan et al. 2004).
Table 1

The Number of Optical Matches for HICAT

\begin{tabular}{lc}
\hline \hline & Number of Sources \\
\hline Total & 4315 \\
\hline No optical match & 596 \\
Position and velocity match & 3313 \\
Position match & 406 \\
\hline Bonne et al. (2015) & 1043 \\
NED and HYPERLEDA & 2270 \\
HOPCAT & 406 \\
\hline
\end{tabular}

For each HI source in HICAT, we search for optical counterparts using the position and velocity measurements in HICAT. Since the HIPASS data has a spatial resolution of $15 ! 5$, it is necessary to obtain more accurate positions for the $\mathrm{H}$ I sources before searching for their mid-infrared counterparts in the WISE frames. To this end, we search for optical counterparts in the spectroscopic sample of Bonne et al. (2015), the NASA/IPAC Extragalactic Database (NED), ${ }^{4}$ HYPERLEDA (Makarov et al. 2014), and HOPCAT (Doyle et al. 2005). In order to accurately match the H I sources with their optical counterparts, we cross-check the velocity measurements from HICAT with the velocity provided from the above sources for all possible positional matches. We describe each source below-as well as the matching process-in detail.

In our preliminary search for optical counterparts, we use the spectroscopic sample of Bonne et al. (2015), which covers the full survey area of HICAT and provides velocities (predominantly from the $r_{F} \leqslant 15.60$ 2MASS selected $6 \mathrm{dF}$ Galaxy Survey, 6dFGS; Jones et al. 2009), morphologies, and WISE photometry from the All-Sky public-release archive (Cutri et al. 2013a) for 13325 galaxies. For each source, we select the best match to this sample within $5^{\prime}$ and $400 \mathrm{~km} \mathrm{~s}^{-1}$ of the respective HICAT position and velocity, resulting in 1043 optical counterparts of $4315 \mathrm{HI}$ sources.

To obtain additional optical counterparts, we then search in NED and HYPERLEDA for galaxies within $10^{\prime}$ and $400 \mathrm{~km} \mathrm{~s}^{-1}$ of their respective HICAT positions and velocities. As NED and HYPERLEDA draw information from catalogs such as HICAT, it must be ensured that the velocities extracted from these databases are sourced from optical or highresolution $\mathrm{H}$ I radio observations and not sourced from HICAT, and hence that HICAT velocities are not being cross-matched to themselves.

HOPCAT (Doyle et al. 2005) is used as our last source for optical matches. The matches from HOPCAT cannot be crosschecked with known velocities, but are categorized as "good guesses" by Doyle et al. (2005; see the reference for details on HOPCAT and its matching criteria). We do not use HOPCAT as our primary source for optical matches because Bonne et al. (2015), NED, and HYPERLEDA described above provide more recent velocity measurements from 6dFGS and other surveys that were not available at the time HOPCAT was compiled. The final number of galaxies taken from each source described here is listed in Table 1. In total, optical counterparts were obtained for 3719 H I sources. However, 147 of these

\footnotetext{
4 The NASA/IPAC Extragalactic Database (NED) is operated by the Jet Propulsion Laboratory, California Institute of Technology, under contract with the National Aeronautics and Space Administration.
} 
were found to be multi-galaxy systems and were thus excluded from our analysis.

\subsection{WISE Photometry}

WISE was launched in 2009 December, and mapped the entire sky in four mid-infrared bands: $W 1, W 2, W 3$, and $W 4$ (3.4, 4.6, 12, and $22 \mu \mathrm{m}$; Wright et al. 2010). Once WISE depleted its cryogen in 2010 October, it was then operated in a "warm" state using the two short bands and then placed in hibernation for well over $2 \mathrm{yr}$. As part of the NEOWISE program, WISE was reactivated in 2013 September and continues to observe in the $W 1$ and $W 2$ bands (Mainzer et al. 2014). The point source sensitivities of $W 1, W 2, W 3$, and $W 4$ in Vega magnitudes are 16.5, 15.5, 11.2, and 7.9, respectively (Wright et al. 2010), in which W4 is approximately two orders of magnitude more sensitive than the IRAS.

We have measured new WISE photometry for the optical counterparts of the HICAT source using the procedure described in Jarrett et al. (2013). We chose to do this because the profile-fit photometry data in the ALLWISE public-release archive (Cutri et al. 2013a) of the (degraded resolution) mosaics is optimized for point sources (e.g., Jarrett et al. 2013; Cluver et al. 2014, and references therein). Therefore, resolved sources such as our sample galaxies are either measured as several point sources, or their flux is underestimated by the PSF photometry (mpro). The WISE default catalogs do include extended source photometry for 2MASS Extended Source Catalog (2MXSC; Jarrett et al. 2000) galaxies, but the elliptical apertures (gmag) miss a significant fraction of the flux (Cluver et al. 2014).

All measurements are carried out on WISE image mosaics that are constructed from single native frames using a drizzle technique (Jarrett et al. 2012), resampled with 1 sq. arc pixels (relative to a 6 arcsec FWHM beam). Photometry for each individual HIPASS galaxy, principally flux measurements and surface brightness characterizations, are conducted using the system developed by T. H. Jarrett specifically for WISE data (Jarrett et al. 2013, see Section 3.6). The system estimates photometric errors from the formal components, including the sky background variance and the local sky level, instrumental signatures, and the absolute calibration. The error model also takes into account the correlation between resampled pixels through a correction factor, which is detailed in the WISE Explanatory Supplement (see Section 2.3.f, Cutri et al. 2013a). As detailed in Jarrett et al. (2013), the shape (inclination) and orientation were determined at a fixed $3 \sigma$ isophotal level, which provides a robust and relatively accurate $(<5 \%)$ estimate, although this assumes symmetry and a fixed shape to the $1 \sigma$ edge of the galaxy.

As detailed in T. H. Jarrett et al. (2013, 2018, in preparation), isophotal measurements at the $W 11 \sigma$ level typically capture more than $96 \%$ of the total light for bulge-dominated galaxies, and to a lesser extent $(\geqslant 90 \%)$ for late-type galaxies, and most notably in the $W 3$ and $W 4$ bands as much as $20 \%$ of the light can be missing with low surface brightness galaxies. Hence, total fluxes are important in order to estimate the dust-obscured star formation activity in the $W 3$ and $W 4$ bands. The total flux is estimated by fitting a double Sérsic function to the axisymmetric radial profile, consisting of an inner bulge and an outer disk. Integrating the composite Sérsic model from the $1 \sigma$ isophote to the edge of the galaxy (3 disk scale lengths) recovers the light that is below the single-pixel noise threshold;
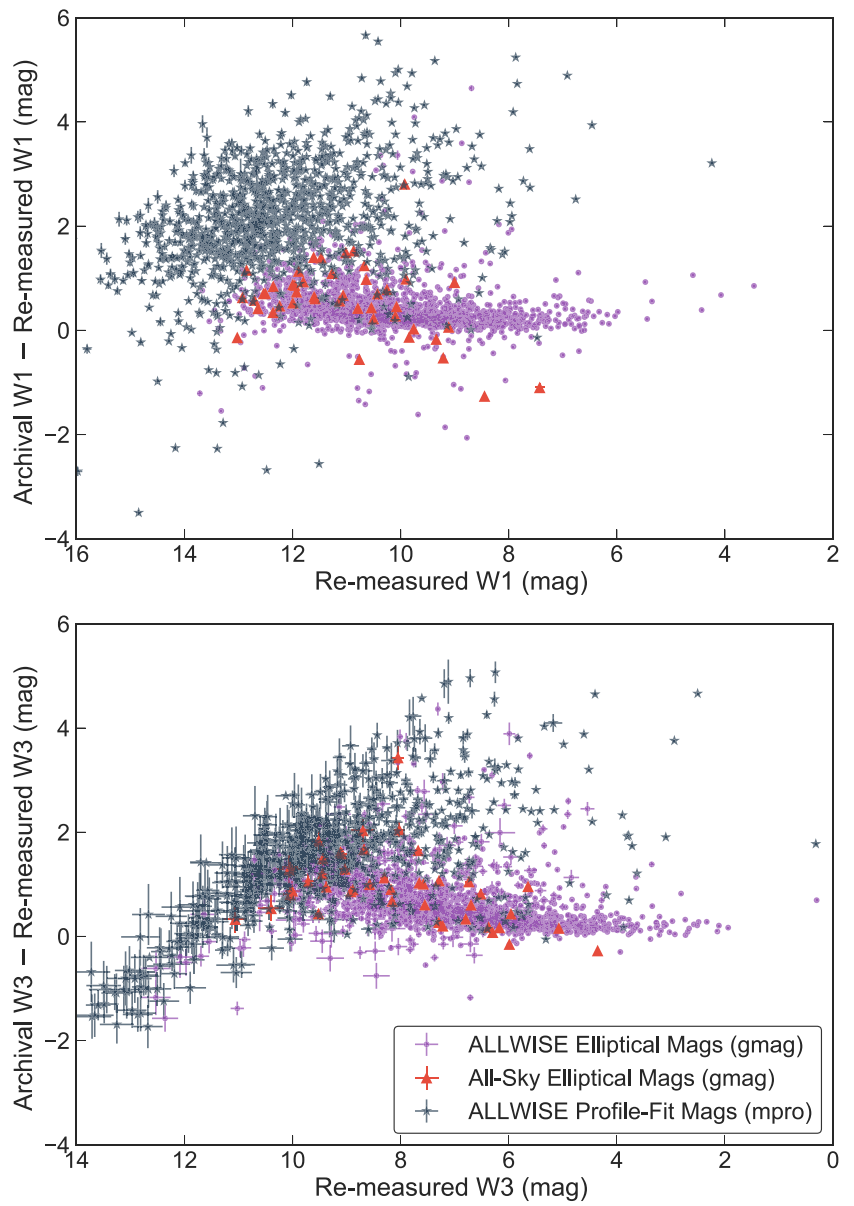

Figure 1. Difference between the archival and new magnitudes as a function of the new magnitude for $W 1$ (top) and $W 3$ (bottom). In order of preference, the archival data are either elliptical aperture magnitudes from the ALLWISE (purple) catalog or All-Sky magnitudes (navy) catalog, or PSF profile-fit (red) magnitudes from the ALLWISE catalog (Cutri et al. 2013b). For galaxies with $W 1 \sim 12 \mathrm{mag}$, the default elliptical aperture photometry is typically in error by $\sim 1 \mathrm{mag}$, while the PSF profile-fit photometry is in error by $\sim 2 \mathrm{mag}$.

details of the fitting process may be found in Jarrett et al. (2013). The error model for the total fluxes includes the goodness of fit, as well as the previous sky estimation per pixel estimates, and typically adds $4 \%-5 \%$ to the isophotal flux uncertainty.

Lastly, each galaxy mosaic is visually inspected, and if necessary, bright stars and nearby galaxies are manually masked out, and the apertures are adjusted. Figure 1 compares our new WISE photometry for HICAT galaxies with the previously available archival fluxes and illustrates the impact of the new photometry on derived quantities. For example, for $W 1 \sim 12$ mag galaxies our $W 1$ and $W 3$ magnitudes are on average systematically brighter by $\sim 1.4$ mag and 1.1 mag than the default photometry pipeline magnitudes. Figure 2 shows four examples of the WISE mosaics that have been cleaned of neighboring objects, as well as the elliptical apertures used for photometry.

We apply an $\mathrm{S} / \mathrm{N}$ threshold of 5 in the $W 1$ and $W 2$ bands, an $\mathrm{S} / \mathrm{N}$ threshold of 3 in the $W 3$ band, and reject confused sources or H I sources consisting of multiple galaxies. Consequently, we measure good $W 1-W 2$ photometry for $3275 \mathrm{H}$ I sources and good W1-W2-W3 photometry for $2831 \mathrm{H}$ I sources. We find 20 $\mathrm{H}$ I sources that do not meet any of our WISE signal-to-noise 

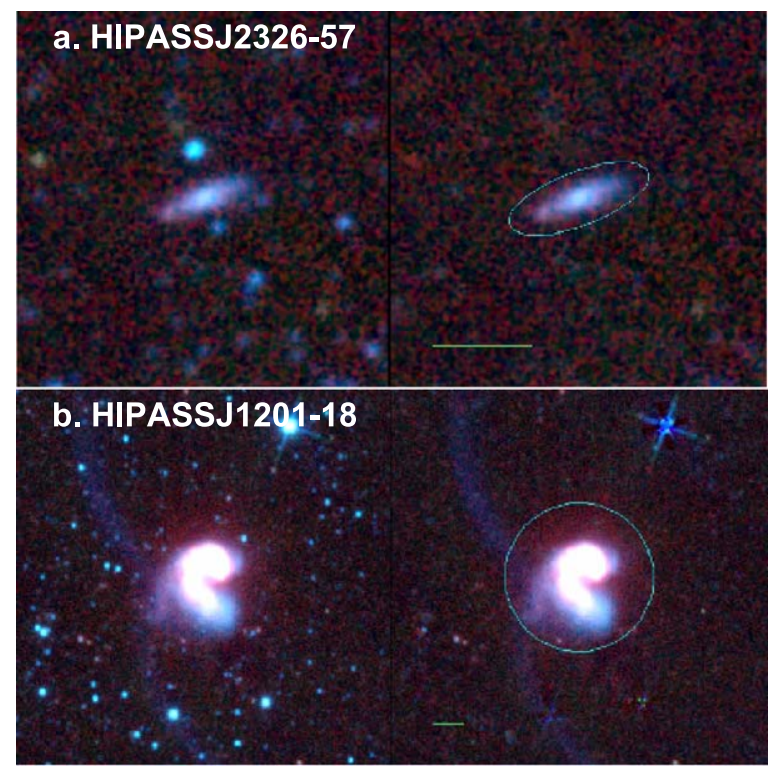

c. HIPASSJ1157-10

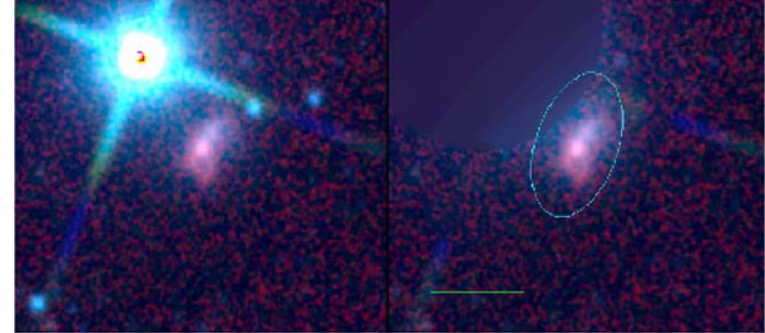

d. HIPASSJ1038-07

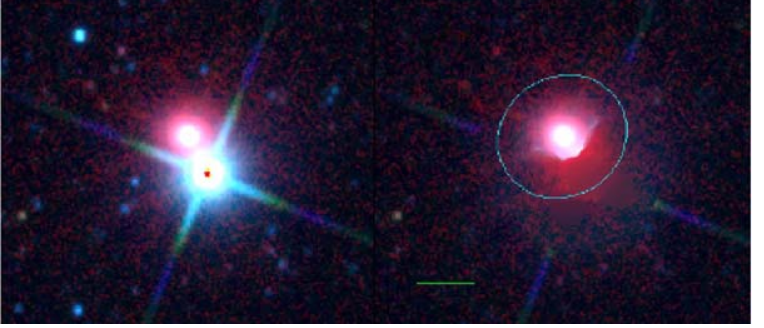

Figure 2. WISE color images composed of $W 1, W 2, W 3, W 4$ bands of four $\mathrm{H} \mathrm{I}$ sources showing the galaxies before (left) and after (right) star and background source removal. The cyan ellipses indicate the $1 \sigma$ apertures. The light from evolved stars is in blue and active star formation is in red. A scale of $2^{\prime}$ is indicated by the green horizontal line. North is upwards and east is to the left. HIPASSJ2326-57 is an example of a well-behaved photometric galaxy; HIPASSJ1201-18 is a multi-galaxy H I source; HIPASSJ1157-10 and HIPASSJ1038-07 are both flagged visually and have a $W 1$ and $W 2 \mathrm{~S} / \mathrm{N}<5$, respectively.

thresholds and $147 \mathrm{H} \mathrm{I}$ sources are multi-galaxy sources. From top to bottom, Figure 2 shows examples from HICAT of a "well-behaved" source, a multi-galaxy source, and two visually flagged sources. A full list of parameters for HICAT+WISE (H I-WISE) is given in Table 8 in the Appendix. The $147 \mathrm{H} \mathrm{I}$ sources that are found to be multi-galaxy systems are excluded from Table 8.

Figure 3 illustrates the WISE colors of HICAT galaxies, along with the expected colors of different types of galaxies, and clearly demonstrates that HIPASS is dominated by star-forming spiral galaxies, with relatively few ellipticals and luminous infrared galaxies (LIRGs). The galaxies with intermediate disk colors in the active galactic nucleus (AGN)/

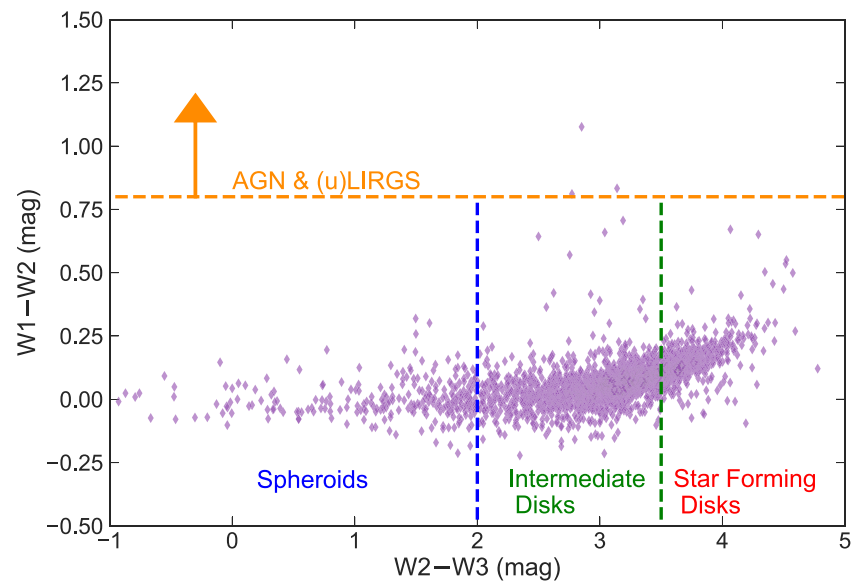

Figure 3. WISE mid-infrared colors of the HICAT sources with good WISE photometry. The horizontal and vertical lines denote the division between different types of galaxies (e.g., Jarrett et al. 2017). HICAT is dominated by star-forming, intermediate, or late-type disk galaxies.

LIRGs region may harbor dust-obscured AGNs and Seyferts (Jarrett et al. 2011; Huang et al. 2017).

\section{Stellar Mass, H I Mass, and SFR Estimation}

In this section, we will describe the methods used to estimate stellar mass, H I mass, and SFR for our samples. For these quantities, we compiled distances from (in order of preference) Cosmicflows-3 (Tully et al. 2016) and HICAT. When distances are not available in the Cosmicflows-3 database, we determine luminosity distances using HIPASS redshifts by applying a cosmic microwave background (CMB)-frame correction using the CMB dipole model presented by Fixsen et al. (1996), followed by an application of the LCDM. For samples selected by stellar mass (Sections 4.2 and 4.3), we include some galaxies that are not in HICAT, and for these galaxies, we use luminosity distances from velocities reported by NED in the same manner as stated above when distances are not available in the Cosmicflows- 3 database. Table 8 lists these distances - and their respective sources-for each galaxy.

\subsection{Stellar Mass}

The $W 1$ and $W 2$ bands are dominated by light from $\mathrm{K}$ - and M-type giant stars, and thus trace the continuum emission from evolved stars with minimal extinction at low redshifts. Consequently, these bands are good tracers of the underlying stellar mass of a galaxy (Meidt et al. 2012; Cluver et al. 2014). However, the $W 2$ band is also sensitive to hot dust, as well as $3.3 \mu \mathrm{m}$ polycyclic aromatic hydrocarbon (PAH) emission from extreme star formation and AGNs. $W 1$ contains the $3.3 \mu \mathrm{m}$ $\mathrm{PAH}$ emission, but it is typically weak for normal star-forming galaxies (Ponomareva et al. 2018). As such, the aggregate stellar mass will be overestimated in the presence of an AGN (Jarrett et al. 2011; Stern et al. 2012; Meidt et al. 2014). In order to mitigate this problem, we exclude AGNs from our analysis that are identified in the AGN catalog of Véron-Cetty \& Véron (2010).

Stellar masses were estimated following the GAMA-derived stellar mass-to-light ratio $\left(M_{*} / L_{W 1_{\text {Sun }}}\right)$ relation of Cluver et al. (2014):

$$
\log _{10} M_{*} / L_{W 1_{\text {Sun }}}=-1.96(W 1-W 2)-0.03,
$$


which depends on the "in-band" luminosity relative to the Sun,

$$
L_{W 1_{\text {Sun }}}=10^{-0.4\left(M-M_{\text {Sun }}\right)} \text {, }
$$

where $M$ is the absolute $W 1$ magnitude and $M_{\text {Sun }}=3.24$ (Jarrett et al. 2013). Equation (1) was determined using the Galaxy and Mass (GAMA; Driver et al. 2009) survey with stellar masses derived assuming a Chabrier (2003) IMF (Taylor et al. 2011; Cluver et al. 2014), and limited to galaxies with $-0.05<$ $W 1-W 2<0.30$, so we restrict the input colors for Equation (1) accordingly. The $W 1-W 2$ color dependence takes into account morphological dependence on the $\mathrm{M} / \mathrm{L}$ and other factors, such as metallicity (Cluver et al. 2014). To determine the $W 1-W 2$ color, apertures are matched between the two bands. We typically use the $W 2$ elliptical isophotal aperture as the fiducial since it is less sensitive than $W 1$ and usually $10 \%$ to $15 \%$ smaller in radial extent. This is particularly the case for galaxies whose mid-infrared emission is dominated by stellar light (see Cluver et al. 2017; Jarrett et al. 2017).

The consistency of our stellar masses and those from other studies can be tested using the MPA-JHU catalog (Brinchmann et al. 2004) $)^{5}$ for the Sloan Digital Sky Survey (SDSS; York et al. 2000). By construction, our WISE stellar masses agree with the SED stellar masses determined by GAMA (Driver et al. 2009; Taylor et al. 2011), with a $1 \sigma$ scatter of just 0.2 dex, and the GAMA $M_{*} / L$ agree with the MPA-JHU $M_{*} / L$, with a bi-weight mean and $1 \sigma$ scatter in the difference between $M_{*} / L$ of -0.01 and 0.07 dex. For dwarf galaxies the difference MPAJHU between and UV-optical SED stellar masses (Huang et al. 2012) is zero with the objects lying within \pm 0.35 dex $M_{\odot}$ (Maddox et al. 2015, and private correspondence). Thus, we do not expect large offsets between the stellar masses we have obtained from WISE and those which have been used in recent studies of H I galaxies.

\subsection{H I Mass}

To calculate the H I mass, we use the published integrated $21 \mathrm{~cm}$ flux $\left(F_{\mathrm{H} \mathrm{I}}\right)$ as follows:

$$
M_{\mathrm{H} \text { I }}\left[M_{\odot}\right]=\frac{2.356 \times 10^{5}}{1+z} \times D_{L}^{2} \times F_{\mathrm{H} \text { I }}\left[\mathrm{Jy} \mathrm{km} \mathrm{s}^{-1}\right] \text {, }
$$

where $D_{L}$ is the luminosity distance to the galaxy in megaparsecs and $z$ is the redshift measured from the $\mathrm{HI}$ spectrum (e.g., Lutz et al. 2017). The uncertainty in the H I mass $\left(\Delta M_{\mathrm{HI}}\right)$ is estimated using the method suggested by Doyle \& Drinkwater (2006):

$$
\begin{aligned}
\Delta F_{\mathrm{HI}} & =0.5 \times F_{\mathrm{HI}^{\prime}}^{1 / 2}, \\
\Delta M_{\mathrm{HI}} & =M_{\mathrm{H}} \frac{\Delta F_{\mathrm{HI}}}{F_{\mathrm{HI}}} .
\end{aligned}
$$

\subsection{Star Formation Rate}

The $W 3$ and $W 4$ bands are sensitive to the interstellar medium, AGNs, and star formation (e.g., Calzetti et al. 2007; Jarrett et al. 2011; Cluver et al. 2014, 2017). W4 emission is dominated by warm dust, and for star-forming galaxies W4 luminosity can be used to predict Balmer-decrement-corrected $\mathrm{H} \alpha$ luminosity with an accuracy of 0.2 dex (Brown et al. 2017).

\footnotetext{
5 Available via http://www.mpa-garching.mpg.de/SDSS/.
}

However, W4 lacks sensitivity and $39 \%$ of HICAT sources lack $W 4$ detections.

WISE W3 luminosity includes contributions from PAHs, nebular emission lines, silicate absorption, and warm dust, all of which are associated with star formation in galaxies. For $\sim L^{*}$ star-forming galaxies, Cluver et al. (2017) found that PAHs and warm dust make $34 \%$ and $62.5 \%$ contributions, respectively, to the observed $W 3$ luminosity. That said, we expect the contribution of PAHs to the W3 luminosity to decrease with decreasing galaxy mass due to the massmetallicity relation of galaxies. Also, W3 better predicts the total infrared luminosity (and hence SFR) than W4 (Cluver et al. 2017). WISE W3 is thus a good SFR indicator, and can be used to obtain the Balmer-decrement-corrected $\mathrm{H} \alpha$ luminosity with an accuracy of 0.28 dex (i.e., Brown et al. 2017).

Although the $W 3$ band traces emission from star formation, it may also have contributions from evolved stellar populations. For $\sim L^{*}$ galaxies located at the center of the star-forming main sequence, the stellar continuum contributes $15.8 \%$ of the W3 light and we subtract it from our data using the $W 1$ photometry and the method of Helou et al. (2004). To account for this, we therefore scale the $W 1$ integrated flux density, and subtract it from the $W 3$ total flux to give an estimate of the $W 3$ emission from the ISM, $W 3_{\text {PAH }}$ (Cluver et al. 2017). We use the prescription in Table 4 from Brown et al. (2017) to estimate the Balmer-decrement-corrected $\mathrm{H} \alpha\left(L_{\mathrm{H}_{\alpha}}\right.$, Corr $)$ :

$$
\begin{aligned}
\log L_{W 3_{\mathrm{PAH}}}\left[\mathrm{erg} \mathrm{s}^{-1}\right]= & (40.79 \pm 0.06)+(1.27 \pm 0.04) \\
& \times\left(\log L_{\mathrm{H}_{\alpha}, \text { Corr }}\left[\mathrm{erg} \mathrm{s}^{-1}\right]-40\right),
\end{aligned}
$$

with a $1 \sigma$ scatter of 0.28 dex. SFRs are estimated by scaling the Kennicutt (1998) calibration to a Chabrier (2003) IMF:

$$
\operatorname{SFR}\left[M_{\odot} \mathrm{yr}^{-1}\right]=\left(4.6 \times 10^{-42}\right) \times L_{\mathrm{H}_{\alpha}} \text { Corr }\left[\mathrm{erg} \mathrm{s}^{-1}\right] .
$$

The uncertainty in SFR is dominated by the scatter in the relationship between WISE W3 luminosity and Balmerdecrement-corrected $\mathrm{H} \alpha$ luminosity and therefore the uncertainty in $\log (\mathrm{SFR}) \sim 0.28$ dex (Brown et al. 2017).

We use the SFR calibration from Brown et al. (2017) because it provides better SFR estimates for a broad range of galaxies-including LIRGs and blue compact dwarfs galaxies - compared to the prior literature. Also, Cluver et al. (2017) compared the SFR calibrations from the prior literature to their calibration derived from total infrared luminosity and found the SFR calibration from Brown et al. (2017) to agree with their own.

\section{Samples}

We create three samples to address specific science questions: an H I-selected sample (H I sample), a stellar massselected sample ( $M_{s}$ sample), and a spiral sample. In addition to addressing specific science questions, these samples allow us to compare to the prior literature and to explore the impact that galaxy morphology and selection bias have on the scaling relationships between star formation, stellar mass, and H I mass.

For the remainder of the paper we focus on galaxies (including 3513 HICAT galaxies) that are at least $10^{\circ}$ away from the Galactic plane and are not known AGNs (from the Véron-Cetty \& Véron (2010) catalog), although we do include some additional galaxies in our summary of galaxy coordinates, redshifts, and photometry provided in Table 8 . We also 


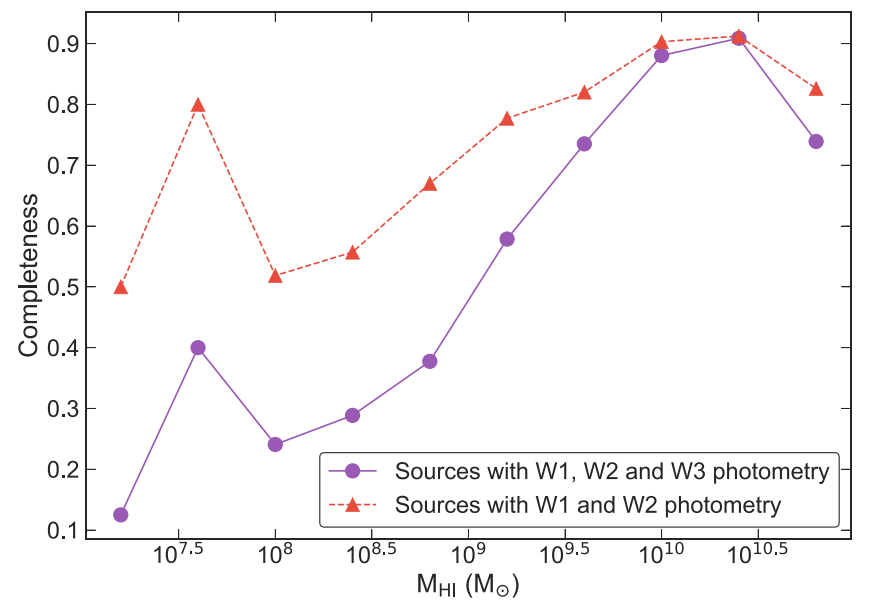

Figure 4. Fraction of HICAT sources with WISE photometry, compared to the total sample, as a function of H I mass. Out of 3513 HICAT sources, $80 \%$ have good $W 1$ and $W 2$ detections and $68 \%$ have good $W 3$ detections.

exclude objects north of the main HICAT footprint $(\delta \geqslant+2)$ from the $M_{s}$ sample and spiral sample.

\subsection{H I-selected Sample}

We use HICAT to form the basis of the H I-selected sample. Out of 3513 HICAT sources, 2826 have good $W 1$ and $W 2$ photometry. Of these, 2396 also have good photometry for the W3 band, and 2342 galaxies in this category have significant $W 3_{\text {PAH }}$ flux ( $W 3$ flux with the stellar continuum subtracted). Figure 4 shows the percentage of HI sources with WISE counterparts as a function of H I mass. We achieve a HICATWISE match completeness of $80 \%$ for $\mathrm{HI}$ mass $>10^{9.5} \mathrm{M}_{\odot}$.

\subsection{Spiral Sample}

To measure the distribution of $\mathrm{HI}$ masses and SFRs as a function of stellar mass and morphology, we generate a stellar mass-selected sample of spiral galaxies (the spiral sample). The spiral sample is drawn from the Bonne et al. (2015) catalog, which achieves a $99 \%$ completeness in redshifts and morphologies for galaxies with $K_{\text {tot }}<10.75$. We maximize H I completeness by limiting the sample to spiral galaxies (defined with de Vaucouleurs T-type $\geqslant 0$ ) with redshift $\leqslant 0.01$ and remeasured $W 1$ magnitude $<10$. Our spiral sample consists of 600 galaxies.

For 435 of our spiral galaxies we obtained H I fluxes from HICAT, while for a further 121 galaxies we obtained archival H I fluxes from Paturel et al. (2003), Huchtmeier \& Richter (1989), Springob et al. (2005), and Masters et al. (2014). The details of the H I counterparts are provided in Table 2. As we illustrate in Figure 5,93\% of the spiral galaxies have H I detections.

The aforementioned $W 1$ and redshift limits are chosen because of the brightness limitation of the parent sample and the detection sensitivity of HICAT. While the notional limit for the Bonne et al. (2015) is $K_{\text {tot }} \leqslant 10.75$, this limits the $W 1 \leqslant$ 11.5 and galaxy numbers decline at $W 1>10$. Table 3 lists the number of galaxies in our sample, the number with $\mathrm{HI}$ detections and the percentage with $\mathrm{HI}$ detections with and without the redshift and $W 1$ magnitude limits applied. Removing the redshift and magnitude limits from the spiral sample decreases the percentage of galaxies with $\mathrm{H}$ I detections to $50 \%$, but has little impact on measured relations we describe in Section 5.

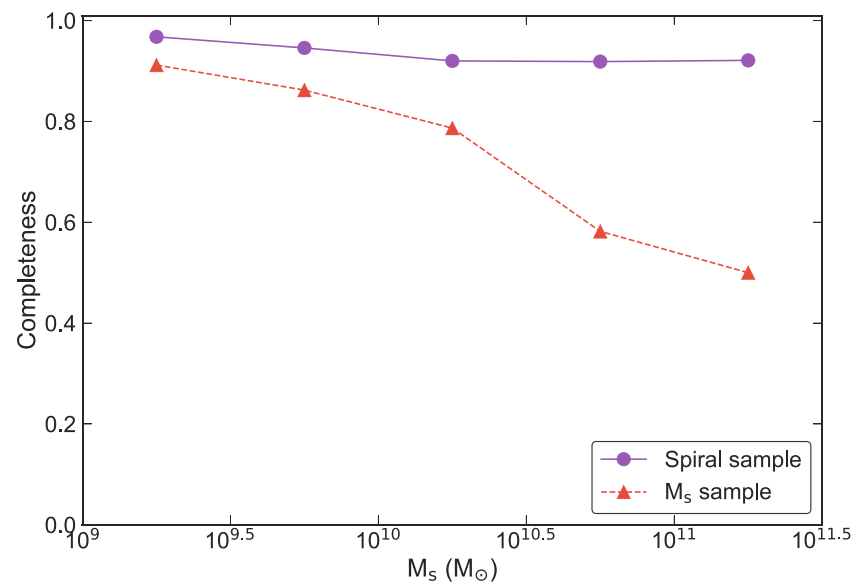

Figure 5. Fraction of galaxies in the spiral sample and the $M_{s}$ sample with $\mathrm{H} \mathrm{I}$ mass measurements. For the spiral sample, 556 out of 600 galaxies have a $\mathrm{H} \mathrm{I}$ counterpart, giving the spiral sample a completeness of $93 \% .590$ galaxies out of the 839 galaxies in the $M_{s}$ sample have a H I counterpart.

Table 2

The Number of Galaxies the Spiral Sample and the $M_{s}$ Sample with H I Counterparts

\begin{tabular}{lcc}
\hline \hline H I Source & Spiral Sample & $M_{s}$ Sample \\
\hline Total & 600 & 839 \\
HICAT & 435 & 454 \\
Paturel et al. (2003) & 100 & 114 \\
Huchtmeier \& Richter (1989) & 13 & 13 \\
Springob et al. (2005) & 1 & 2 \\
Masters et al. (2014) & 7 & 7 \\
\hline
\end{tabular}

\subsection{Stellar Mass-selected Sample ( $M_{s}$ Sample)}

In order to compare the HI mass, stellar mass, and SFR relationships of this work to those of GASS (Catinella et al. 2010, 2012, 2013), which uses a stellar mass-selected sample, we have also produced such a sample $\left(M_{s}\right.$ sample). The GASS sample is designed to measure the neutral hydrogen content of 1000 galaxies to investigate the physical mechanisms that regulate how cold gas responds to different physical conditions in the galaxy and the processes responsible for the transition between starforming spirals and passive ellipticals (Catinella et al. 2010).

The $M_{s}$ sample selection is identical to that of the spiral sample except that it lacks the T-type criterion. The $M_{s}$ sample contains 839 galaxies, of which 590 have an H I counterpart. The details of the H I counterparts are provided in Table 2. Figure 5 shows that the fraction of galaxies with an $\mathrm{HI}$ measurement drops at the higher stellar masses, where the number of H I-poor ellipticals increases.

\section{5. $M_{\mathrm{H}}-M_{*}$ Relationship}

In this section, we will look at the relationship between $\mathrm{H} \mathrm{I}$ mass and stellar mass for all three samples. The H I-stellar mass relation is one of the principal means used to provide insight to the history of gas accretion and star formation. Also, as discussed in Section 7, it can be used to test models of the stability of $\mathrm{HI}$ disks and how these disks can fuel star formation (Obreschkow et al. 2016; Wong et al. 2016). For each sample, we bin the data into stellar mass bins with a width of $\log \left(M_{*}\right)=0.5$ and for bins with $\geqslant 10$ galaxies we measure the median $\mathrm{H}$ I mass and $1 \sigma$ scatter in $\mathrm{H}$ I mass for each stellar 
Table 3

The Basic Properties of Our Spiral Sample and Spiral Galaxy Samples without the Redshift and W1 Selection Criteria Applied

\begin{tabular}{lcccc}
\hline \hline & Spiral Sample & No W1-mag cut & No Redshift Cut & No W1 Magnitude and Redshift Cuts Applied \\
\hline Total \# of galaxies & 600 & 792 & 1442 & 3458 \\
\# H I counterparts & 556 & 672 & 1048 & 1697 \\
\% with H I counterpart & $93 \%$ & $87 \%$ & $73 \%$ & $50 \%$ \\
\hline
\end{tabular}

Table 4

Median H I Mass and $1 \sigma(68 \%)$ Scatter as a Function of Stellar Mass for the Spiral Sample, and the GASS Spiral Sample

\begin{tabular}{|c|c|c|c|c|c|c|c|c|}
\hline \multirow[b]{2}{*}{$\begin{array}{l}\log \left(M_{*}\right) \\
\left(M_{\odot}\right)\end{array}$} & \multicolumn{4}{|c|}{ Spiral Sample } & \multicolumn{4}{|c|}{ GASS Spiral Sample } \\
\hline & $\begin{array}{c}\log \left(M_{\mathrm{HI}}\right) \\
\left(M_{\odot}\right)\end{array}$ & $\begin{array}{c}1 \sigma \\
\left(M_{\odot}\right)\end{array}$ & Total & $\begin{array}{c}\% \mathrm{H} \mathrm{I} \\
\text { Detections }\end{array}$ & $\begin{array}{c}\log \left(M_{\mathrm{HI}}\right) \\
\left(M_{\odot}\right)\end{array}$ & $\begin{array}{c}1 \sigma \\
\left(M_{\odot}\right)\end{array}$ & Total & $\begin{array}{c}\% \mathrm{H} \mathrm{I} \\
\text { Detections }\end{array}$ \\
\hline 9.75 & 9.41 & 0.37 & 129 & 95 & & & & \\
\hline 10.25 & 9.59 & 0.43 & 225 & 92 & 9.53 & 0.47 & 130 & 89 \\
\hline 10.75 & 9.61 & 0.46 & 174 & 97 & 9.73 & 0.38 & 111 & 94 \\
\hline 11.25 & 9.93 & 0.54 & 38 & 92 & 9.88 & 0.55 & 64 & 83 \\
\hline
\end{tabular}

Note. Median H I masses are not calculated for stellar mass bins with a H I completeness $\leqslant 50 \%$.

mass bin. The $1 \sigma$ scatter about the median is determined using the range encompassing $68 \%$ of the data. For the spiral sample and $M_{s}$ sample, care is needed when accounting for the galaxies with $\mathrm{H}$ I nondetections. When estimating the median $\mathrm{H}$ I mass as a function of the stellar mass, we assume the $\mathrm{H} \mathrm{I}$ upper limits are below the median H I mass for the relevant stellar mass bin, which is a reasonable approximation when the $\mathrm{HI}$ detection rate is $\gg 50 \%$. The median $\mathrm{HI}$ masses are determined for any stellar mass bin with 10 or more galaxies and $\mathrm{HI}$ detection rate above $50 \%$. Upper limits for individual galaxies are determined using the integrated flux of 7.5 Jy km s${ }^{-1}$, corresponding to the HICAT's 95\% completeness limit (Zwaan et al. 2004).

While we list the individual uncertainties in Table 8, we find that $W 1 \sim 12$ galaxies have a stellar mass uncertainty $\leqslant 0.2$ dex, and this uncertainty decreases with increasing $W 1$ flux. Also, $80 \%$ of the H I sample has an H I mass uncertainty better than $20 \%$.

\subsection{H I Sample}

The relationship between H I mass and stellar mass for the $\mathrm{H}$ I-selected sample is illustrated in Figure 6. The H I mass is a strong function of stellar mass among the H I sample (Spearman's rank correlation, $r_{s},=0.64$ ) and the least-squares fit to the medians, represented by an orange line in Figure 6, is

$$
\log M_{\mathrm{H}}=0.51\left(\log M_{*}-10\right)+9.71 .
$$

We fit $68 \%$ of the HI masses within 0.5 dex of our best-fit relation.

$\mathrm{H}$ I mass versus stellar mass relations for the $\mathrm{H}$ I-selected sample and previous studies (Catinella et al. 2010; Huang et al. 2012; Maddox et al. 2015) are also plotted in Figure 6. Our sample and the ALFALFA samples of Huang et al. (2012) and (Maddox et al. 2015) are H I-selected, while the GASS sample of Catinella et al. (2010) is stellar mass-selected. We find the relations for the $\mathrm{HI}$-selected samples are qualitatively similar, with median $\mathrm{HI}$ mass increasing with stellar mass. In contrast, the H I versus stellar mass relation measured with the GASS sample (Catinella et al. 2010 ) is up to 0.5 dex lower than those derived from H I-selected samples, as the GASS sample includes galaxies with low H I masses (including ellipticals). There are also discrepancies in the $\mathrm{HI}$ versus stellar mass relations measured with different $\mathrm{HI}$ selected samples. We do not see the break in the relation at a stellar mass of $10^{9} M_{\odot}$ that was previously observed by ALFALFA (i.e., Huang et al. 2012; Maddox et al. 2015). ${ }^{6}$ Below a mass of $10^{9} M_{\odot}$ our sample is less than $70 \%$ complete for WISE counterparts, and thus we may not be reliably measuring $\mathrm{H}$ I mass versus stellar mass in this mass range. However, even if this was not an issue we believe this sample would produce a biased relation, as it (by construction) excludes galaxies that have high stellar masses but low H I masses (i.e., many elliptical galaxies).

\subsection{Spiral Sample}

The H I and stellar mass distribution for the spiral sample is shown in Figure 7. The median H I mass increases with stellar mass with a least-squared fit of

$$
\log M_{\mathrm{H}}=0.35\left(\log M_{*}-10\right)+9.45,
$$

with $68 \%$ of the H I masses within 0.4 dex.

However, as Figure 7 illustrates, at a given stellar mass the median HI mass increases with T-type while the dispersion decreases with T-type. For example, for the $10^{10}$ to $10^{10.5} M_{\odot}$ stellar mass bin, the median H I mass and the $1 \sigma$ spread of galaxies for all spirals is $10^{9.53} M_{\odot} \pm 0.47 \mathrm{dex}$, for T-type 0 to 2 is $10^{9.26} M_{\odot} \pm 0.59 \mathrm{dex}$, and for T-type 6 to 8 is $10^{9.72}$ $M_{\odot} \pm 0.31$ dex. The increasing spread of $\mathrm{HI}$ masses with decreasing T-type for spiral galaxies may be part of a broader trend, as Serra et al. (2012) concluded that the HI mass distribution for early-type galaxies was far broader than that for spirals. They suggest this scatter reflects the large variety of $\mathrm{H} \mathrm{I}$ content of early-type galaxies, and confirms the lack of correlation between H I mass and luminosity.

In Figure 7, we compare our $\mathrm{HI}$ mass-stellar mass distribution of our spiral sample to that from GASS (Catinella

\footnotetext{
6 The slopes of the least-squared fits for Figure 6 is $0.65 \pm 0.014$ for stellar masses $\leqslant 10^{9} M_{\odot}$ and $0.48 \pm 0.013$ for stellar mass $>10^{9} M_{\odot}$ and therefore are consistent with each other. Meanwhile, Huang et al. (2012) measured a slope of 0.712 for stellar masses $\leqslant 10^{9} M_{\odot}$ and 0.276 for stellar masses $>10^{9} M_{\odot}$.
} 


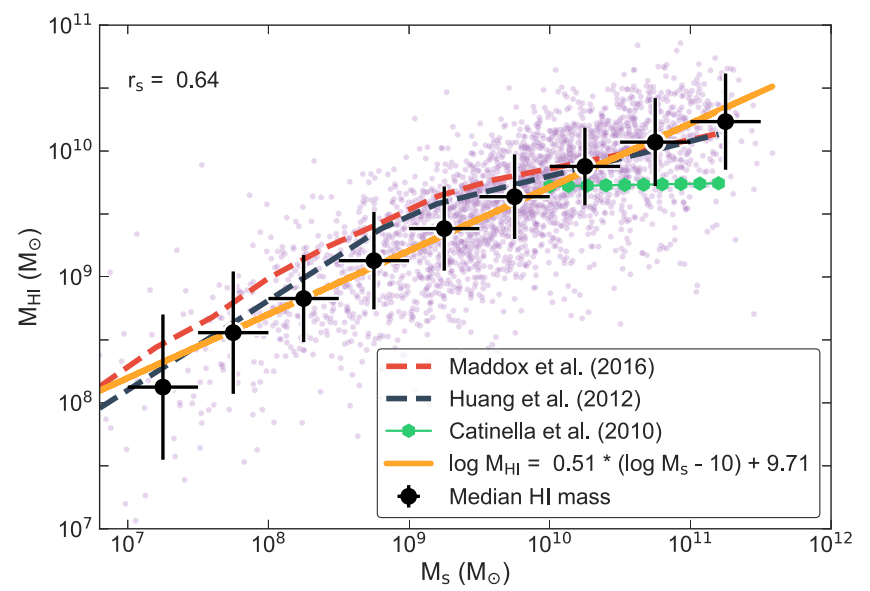

Figure 6. H I mass vs. stellar mass of the H I-selected sample. Spearman's rank correlation, $r_{s}$, is listed in the top left corner. Our median $\mathrm{H}$ I masses are slightly below those derived by Huang et al. (2012; blue dashed line), and Maddox et al. (2015; red dashed line), who both used ALFALFA H I-selected samples. However the H I mass medians of the H I sample are higher than the GASS sample (green solid-line; Catinella et al. 2010), which is a stellar mass-selected sample. H I samples overestimate H I mass as a function of stellar mass because $\mathrm{H}$ I samples do not detect galaxies with low $\mathrm{H}$ I mass, such as ellipticals.

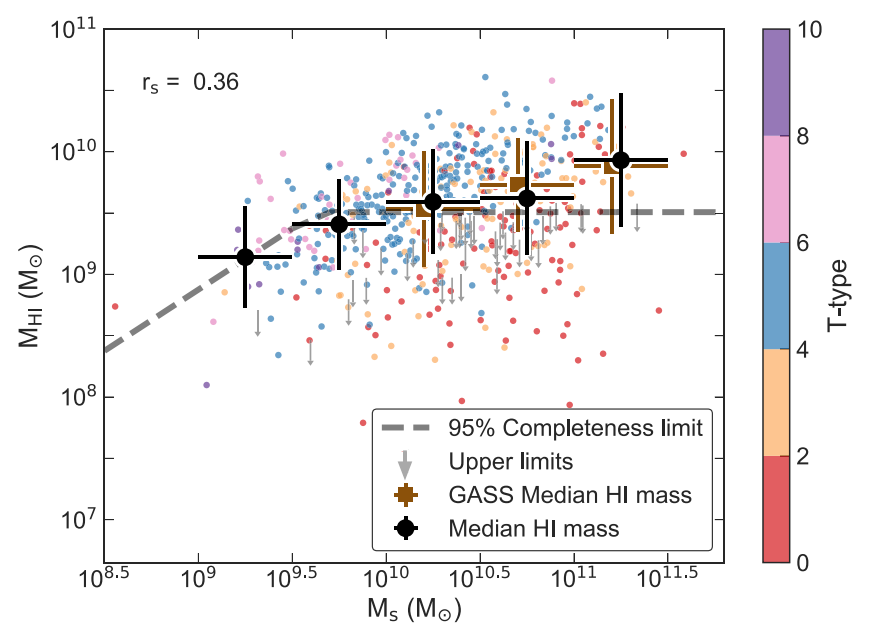

Figure 7. H I mass vs. stellar mass for the (highly complete) spiral sample. Symbols are color-coded by morphology, the median H I masses are shown for the spiral sample (black circles) and the GASS sample (brown squares; Catinella et al. 2010). The dashed line shows the H I mass limit for $z \leqslant 0.01$ and $W 1 \leqslant 10$ star-forming galaxies. The Spearman's rank correlation coefficient is shown in the the top left. H I mass is a function of both T-type and stellar mass. The median H I masses increases with stellar mass with a power-law index of 0.35 .

et al. 2010), using GASS galaxies that we have classified as spirals with Galaxy Zoo 1 (GZ1; Lintott et al. 2011). Using the GZ1 classifications and a $70 \%$ vote threshold, we find GASS comprises 305 spirals, 273 ellipticals, and 182 galaxies with uncertain morphology. ${ }^{7}$ We repeat our analysis on the GASS sample, using the same stellar mass bins and H I median mass calculations with stellar mass bins with 10 or more galaxies and an $\mathrm{H}$ I detection rate $>50 \%$. The estimated median $\mathrm{H}$ I masses

\footnotetext{
7 Using the default criteria of $80 \%$ vote threshold (see the following references for details on Galaxy Zoo and the data release: Lintott et al. 2008, 2011), 291 galaxies (39\%) in GASS are classified as unknowns. We have decreased the vote requirement to $70 \%$ to decrease the number of unknowns to 182 galaxies (24\%), although we find this has little impact on our measured relations.
}

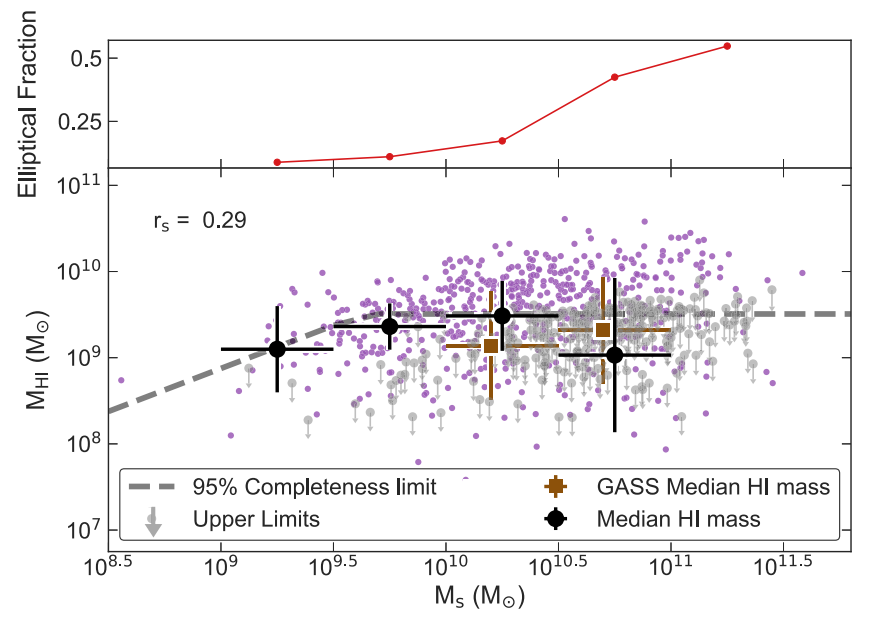

Figure 8. Spiral fraction vs. stellar mass (upper panel) and H I mass vs. stellar mass (lower panel) for the $M_{S}$ sample. Median H I masses are shown for the $M_{s}$ sample (black circles) and the GASS sample (brown squares; Catinella et al. 2010). The HIPASS mass limit for galaxies at $z \leqslant 0.01$ and $W 1 \leqslant 10$ is shown by the gray dashed line and the Spearman's rank correlation coefficient is shown in the top left. H I mass is almost flat with increasing stellar mass for both samples, in large part because of the increasing fraction of passive earlytype galaxies with increasing stellar mass.

for the spiral sample and the GASS spiral samples are listed in Table 4. The median H I masses for the GASS spiral sample increases with stellar mass similar to our spiral sample. The median H I mass of the spiral samples differs by about $0.08 \mathrm{dex}$ on average. To explain the relationship between H I mass and stellar mass of the spiral samples we turn to the halo spin parameter models of Obreschkow et al. (2016) in Section 7.

\section{3. $M_{\mathrm{s}}$ Sample}

In Figure 8, we present H I mass versus stellar mass for our $M_{s}$ sample and the equivalent stellar mass-selected sample of GASS (Catinella et al. 2010). The estimated median H I masses of the $M_{S}$ sample and the GASS sample are listed in Table 5. For the $M_{s}$ sample the H I mass increases with the stellar mass for the stellar mass bins $\leqslant 10^{10.5} M_{*}$ and then flattens for the highest stellar mass bin. The estimated H I mass median for the highest stellar mass bin may be underestimated, as the $\mathrm{HI}$ completeness for this bin is only $58 \%$ and our assumption that all nondetections are below the median could be in error.

At stellar masses greater than $10^{10} M_{\odot}$, median H I mass is almost constant with stellar mass, and our measurements agree with those of GASS to within 0.3 dex. This is in contrast with the trend shown in Figure 7 for the spiral sample. The obvious explanation, given the prior literature (e.g., Catinella et al. 2010; Huang et al. 2012), is that this is due to the increasing fraction of gas poor early-type galaxies at high $M_{*}$, as illustrated in the top panel of Figure 8. Serra et al. (2012) found that early-type galaxies host less H I than spiral galaxies, but have a broader range of H I masses. For example, Serra et al. (2012) found that elliptical galaxies have H I mass from $10^{7}$ to $10^{9} M_{\odot}$ (the lower limit is uncertain as this overlooks H I nondetections in their sample), while the $\mathrm{H}$ I mass distribution for spirals peaks at $\sim 2 \times 10^{9} M_{\odot}$, with a small number of galaxies below $10^{8} M_{\odot}$. Combining this result with our previous findings from our spiral sample, we conclude that as one moves from early-type to late-type galaxies, median $\mathrm{H} \mathrm{I}$ mass increases while the scatter in H I mass decreases. Within an individual T-type, H I mass typically increases with stellar 
Table 5

Median H I Mass and $1 \sigma(68 \%)$ Scatter as a Function of Stellar Mass for the $M_{s}$ Sample and the GASS Sample

\begin{tabular}{|c|c|c|c|c|c|c|c|c|}
\hline \multirow[b]{2}{*}{$\begin{array}{l}\log \left(M_{*}\right) \\
\left(M_{\odot}\right)\end{array}$} & \multicolumn{4}{|c|}{$M_{s}$ Sample } & \multicolumn{4}{|c|}{ GASS Spiral Sample } \\
\hline & $\begin{array}{c}\log \left(M_{\mathrm{HI}}\right) \\
\left(M_{\odot}\right)\end{array}$ & $\begin{array}{c}1 \sigma \\
\left(M_{\odot}\right)\end{array}$ & Total & $\begin{array}{c}\% \mathrm{H} \mathrm{I} \\
\text { Detections }\end{array}$ & $\begin{array}{c}\log \left(M_{\mathrm{HI}}\right) \\
\left(M_{\odot}\right)\end{array}$ & $\begin{array}{c}1 \sigma \\
\left(M_{\odot}\right)\end{array}$ & Total & $\begin{array}{c}\% \mathrm{H} \mathrm{I} \\
\text { Detections }\end{array}$ \\
\hline 9.25 & 9.10 & 0.50 & 34 & 91 & & & & \\
\hline 9.75 & 9.56 & 0.27 & 145 & 86 & & & & \\
\hline 10.25 & 9.48 & 0.41 & 272 & 79 & 9.14 & 0.64 & 299 & 68 \\
\hline 10.75 & 9.03 & 0.89 & 299 & 58 & 9.32 & 0.62 & 292 & 63 \\
\hline 11.25 & & & 84 & 50 & & & 168 & 50 \\
\hline
\end{tabular}

Note. Median H I masses are not calculated for stellar mass bins with an H I completeness $\leqslant 50 \%$.

mass, and it is the increasing fraction of early-types with increasing stellar mass that explains the roughly constant median H I masses measured for the $M_{s}$ sample.

\subsection{The Impact of Sample Selection}

A key conclusion from the previous sections is that sample selection impacts measured HI mass versus stellar mass relations, and, to illustrate this, in Figure 9 we plot H I massstellar mass relations for $\mathrm{H}$ I-selected samples, spiral galaxy samples, and stellar mass-selected samples, including data from both our work and the literature. For all values of stellar mass, $\mathrm{H}$ I-selected samples have a higher $\mathrm{H}$ I mass than spiral-selected and stellar mass-selected samples. This is because H I surveys are designed to sample a large number of $\mathrm{H} \mathrm{I-rich} \mathrm{systems,} \mathrm{and}$ therefore lack the sensitivity to detect the H I-poor galaxy population. For example, HIPASS can detect galaxies with H I masses $>10^{9} M_{\odot}$ at $z=0.01$; however, elliptical galaxies have H I masses $\leqslant 10^{9} M_{\odot}$ (Serra et al. 2012), and would thus be largely missing from HIPASS samples at these redshifts. Even the late-type galaxies in Figure 7 have $\mathrm{H}$ I masses as low as $10^{8}$ $M_{\odot}$, and thus some are missing from HIPASS-selected samples at $z>0.01$. Similar selection effects apply to ALFALFA, albeit at higher redshifts. This is not surprising and indeed was a motivation for studies such as GASS, but does illustrate that H I mass versus stellar mass relations have a strong dependence on sample selection.

\section{Star-forming Properties of the Spiral Sample}

\subsection{Star-forming Main Sequence}

While the relationship between SFR and HI mass is the principal focus of the paper, we are also able to measure the local $(z \leqslant 0.01)$ star-forming main sequence (MS; e.g., Noeske et al. 2007; Rodighiero et al. 2011; Wuyts et al. 2011) using the spiral sample. Measurements of the MS are enhanced by our highly complete sample, our new WISE photometry (which should mitigate aperture bias-see Section 2.2), and our ability to take advantage of a recent calibration of W3 as an SFR indicator that uses large aperture photometry (Brown et al. 2017).

In Figure 10 we present our star-forming main sequence. As expected, the median of the log SFR increases from -0.50 dex for the $10^{9}$ to $10^{9.5} M_{\odot}$ stellar mass bin to 0.14 dex for the $10^{10}$ to $10^{10.5}$ stellar mass bin, with the scatter of individual galaxies about the median being $\sim 0.3$ dex. For stellar mass bins above $10^{10} M_{\odot}$, the median log SFR is roughly constant at 0.14 while the scatter of the individual galaxy SFRs about the median increases from 0.38 to $0.49 \mathrm{dex}$. The changing trend of SFR with increasing stellar mass and the increased dispersion of

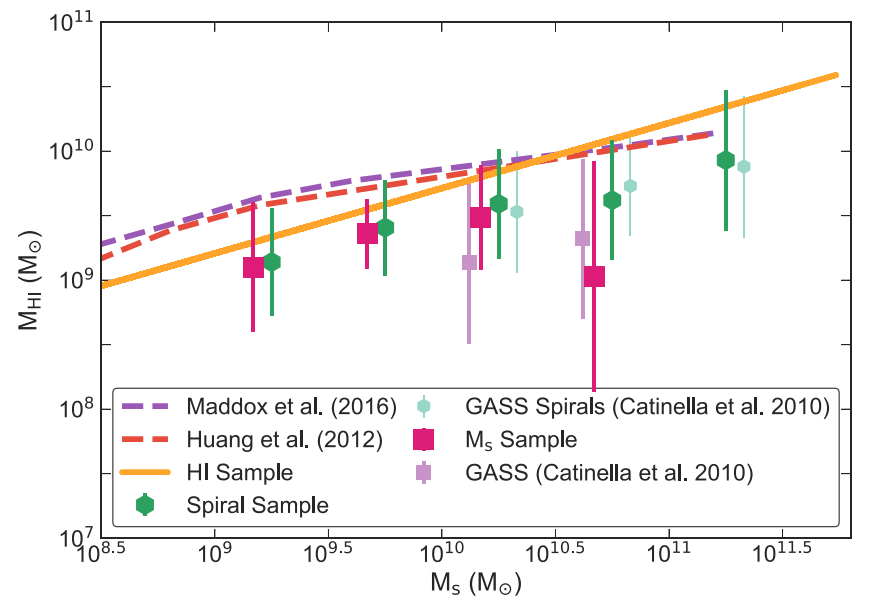

Figure 9. Comparison of $\mathrm{H}$ I mass vs. stellar mass for our $\mathrm{H}$ I-selected sample, the spiral sample, the $M_{s}$ sample (without morphological criteria), and the prior literature. H I-selected samples give consistently higher relations than the other samples, as (by definition) they exclude galaxies with comparatively low H I masses.

SFRs is evidence of mass quenching (Kauffmann et al. 2003), and this also coincides with an increasing fraction of early-type spirals (T-type $\leqslant 2$ ). To mitigate the effect of mass quenching on our model fit to the MS, we only fit to galaxies with stellar mass $\leqslant 10^{10.5} M_{\odot}$ (shown in Figure 10(b)) and measure the MS to be

$$
\log \mathrm{SFR}=0.7\left(\log M_{*}-10\right)-0.09,
$$

with a $1 \sigma$ scatter of 0.27 dex. Alternate selection criteria to mitigate the effect of mass quenching produces similar MS fits. For example, a subsample of galaxies with T-type $>2$ produces a fit of $\log \mathrm{SFR}=0.61\left(\log M_{*}-10\right)-0.08(1 s \sigma$ scatter $=0.26 \mathrm{dex}$.

Figure 10(b) and Table 6 also compare the MS relation from this work to the prior literature (Elbaz et al. 2007; Salim et al. 2007; Chen et al. 2009; Oliver et al. 2010; Zahid et al. 2012; Grootes et al. 2013), with data taken from the extensive review by Speagle et al. (2014). To shift the Speagle et al. (2014) homogenized MS relations from a Kroupa to Chabrier IMF, we apply -0.03 and -0.07 dex shifts to the stellar masses and SFRs, respectively. The blue line is the $z=0.01 \mathrm{MS}$ relation given by Equation (28) from Speagle et al. (2014), and the shaded region is the "true" scatter about the MS (for more details, please refer to Speagle et al. 2014). The normalization (at $\log M_{*}=10$ ) of our best fit is 0.04 dex smaller and the slope is 0.21 larger than the best fit for the MS of Speagle et al. (2014). Speagle et al. (2014) noted that the wide range of the 

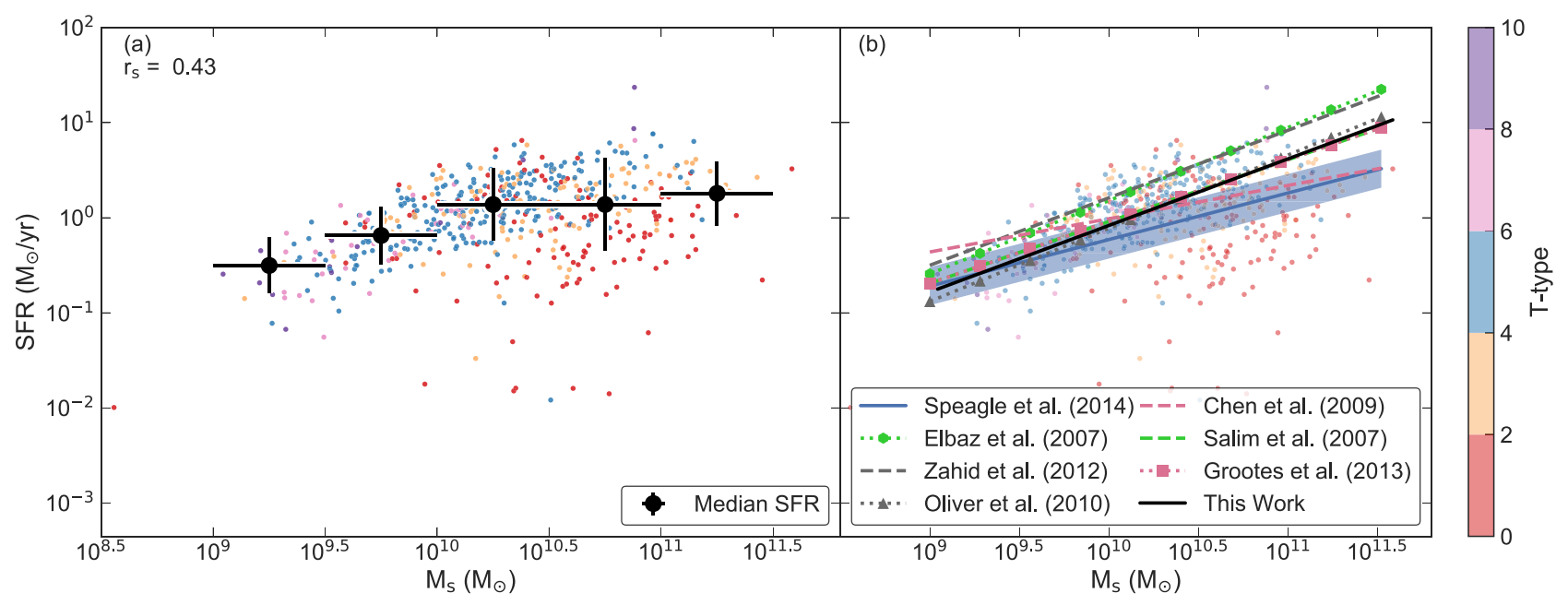

Figure 10. SFR vs. stellar mass for the spiral sample with symbols color-coded by morphology. The median SFRs are also shown in panel (a). The median SFR increases with stellar mass for $M_{*}<10^{10.5} M_{\odot}$ and then flattens for $M_{*}>10^{10.5} M_{\odot}$, indicating evidence of mass quenching. To mitigate the effect of mass quenching, we model the MS using spiral galaxies with $M_{*}<10^{10.5} M_{\odot}$ and find $\log$ SFR $=0.7 \times\left(\log M_{*}-10\right)-0.09$. As illustrated by panel (b), $z \sim 0.01$ relations compiled by Speagle et al. (2014) show considerable scatter, and this may result (in part) from how studies exclude (or include) high-mass spirals that may have already commenced quenching.

MS slopes for the local universe suggests that the systematics involved are underestimated, and they estimate the magnitude of these systematics on the MS slopes to be of the order of $\sim 0.2$ dex. As we noted earlier, our slope depends on the criterion used to reject galaxies that could be undergoing quenching, and including early-type spiral galaxies with masses above $10^{10.5} M_{\odot}$ reduces our slope to $0.416(1 \sigma$ scatter $=0.34)$, which is closer to that of Speagle et al. (2014).

\subsection{Star Formation Efficiency}

SFE, defined as SFR $/ M_{\mathrm{HI}}$, quantifies the current rate of gas consumption, dividing the SFR by HI mass and SFE is expected to depend on the stellar mass of a galaxy (Schiminovich et al. 2010; Huang et al. 2012; Wong et al. 2016; Lutz et al. 2017). SFE and its inverse, the depletion time, have thus been commonly used to quantify gas consumption and test models of the stability of galactic disks (e.g., Wong et al. 2016).

To investigate the relationship between star formation and H I mass within the spiral sample, we plot in Figure 11 SFE as a function of stellar mass. In Table 7 we provide the median SFEs as a function of stellar mass, with the upper limits on the $\mathrm{HI}$ mass being used for the HI nondetections. The SFE remains relatively constant at a median $\mathrm{SFE}=10^{-9.57} \mathrm{yr}^{-1}$, with a $1 \sigma$ scatter of 0.44 dex for spiral galaxies with stellar masses between $10^{9.0}$ and $10^{11.5} M_{\odot}$. While we see evidence for mass quenching in high stellar mass spirals in Figure 10, the SFE appears to be constant for spiral galaxies falling on the MS and spiral galaxies that have (potentially) commenced quenching.

SFE versus stellar mass relations for both the spiral sample and previous studies (Schiminovich et al. 2010; Jaskot et al. 2015; Wong et al. 2016; Lutz et al. 2017) are compared in Figure 12(a). H I-selected samples (Jaskot et al. 2015; Lutz et al. 2017) exhibit an increasing SFE with stellar mass; however, this reflects their selection bias against galaxies with low H I masses. By contrast, stellar mass-selected samples (Schiminovich et al. 2010; Wong et al. 2016) exhibit a constant SFE with stellar mass; however, previous studies have measured differing values of this constant, ranging from $10^{-9.65} \mathrm{yr}^{-1}$ (Wong et al. 2016) to $10^{-9.5} \mathrm{yr}^{-1}$ (Schiminovich et al. 2010). Wong et al. (2016) provide two theoretically motivated relations for SFE versus stellar mass, which are both plotted in Figure 12(b). The first relation assumes that the molecular gas fraction depends only on the stellar surface mass density, while the second assumes that this fraction depends on the hydrostatic pressure. Between stellar masses of $10^{9.0}$ and $10^{11.5} M_{\odot}$, the hydrostatic pressure model of Wong et al. (2016; which gives a constant SFE) shows the greatest consistency with our work and other stellar mass-selected samples.

\section{Discussion}

\subsection{Is there an Upper Limit to the $M_{H_{I}}$ ?}

For the spiral sample, we find that H I mass increases with stellar mass, from $10^{9.14} M_{\odot}$ at a stellar mass of $10^{9.25} M_{\odot}$ to $10^{9.93} M_{\odot}$ at a stellar mass of $10^{11.25} M_{\odot}$ (see Figure 7$)$. We also observe a stellar mass-dependent upper limit on H I mass. In this section, we discuss the reason for this upper limit.

Both our H I- and stellar mass-selected samples imply an upper limit for $\mathrm{H}$ I mass as a function of stellar mass, and such thresholds are also seen in prior literature (e.g., Maddox et al. 2015). Is this upper limit for H I mass expected from theory? Maddox et al. (2015) argue that the maximum H I fraction for galaxies with stellar masses $>10^{9} M_{\odot}$ is set by the upper limit in the halo spin parameter, $\lambda$. The halo spin parameter is defined as

$$
\lambda \equiv J_{\text {halo }} E_{\text {halo }}^{1 / 2} G^{-1} M_{\text {halo }}^{-5 / 2}
$$

where $J_{\text {halo }}$ is the galaxy halo's angular momentum, $E_{\text {halo }}$ its total energy, and $M_{\text {halo }}$ its total mass (Boissier \& Prantzos 2000). Maddox et al. (2015) determined the halo spin parameter of the ALFALFA galaxies and found that, at a fixed stellar mass, galaxies with the largest H I mass also have the largest halo spin parameter (see their Figure 6). The large halo spin of a galaxy stabilizes the high H I mass disk, preventing it from collapsing and forming stars. 
Table 6

Main-sequence Relationships

\begin{tabular}{|c|c|c|c|c|c|c|c|}
\hline Paper & $\alpha$ & $\beta$ & $\log \operatorname{SFR}(10)$ & $z_{\text {med }}$ & $z_{\text {range }}$ & $\log M_{*}$ Range & Survey \\
\hline This Study & 0.7 & -7.09 & -0.09 & $\ldots$ & $\leqslant 0.01$ & $9.0-11.0$ & WISE \\
\hline Zahid et al. (2012) & $0.71 \pm 0.01$ & $-6.78 \pm 0.1$ & 0.32 & 0.07 & $0.04-0.1$ & $8.5-10.4$ & SDSS \\
\hline Oliver et al. (2010) & $0.77 \pm 0.02$ & $-7.88 \pm 0.22$ & -0.18 & 0.1 & $0.0-0.2$ & $9.1-11.6$ & SWIRE \\
\hline Elbaz et al. (2007) & 0.77 & -7.44 & 0.26 & 0.06 & $0.015-0.1$ & $9.1-11.2$ & SDSS \\
\hline Salim et al. (2007) & 0.65 & -6.33 & 0.17 & 0.11 & $0.005-0.22$ & $9.0-11.1$ & GALEX-SDSS selected \\
\hline Speagle et al. (2014) & 0.49 & -5.13 & -0.03 & $\cdots$ & $\ldots$ & $\ldots$ & $\ldots$ \\
\hline
\end{tabular}

Notes. Column 1: reference. Columns 2 and 3: MS slope $\alpha$ and normalization $\beta$ reported in Table 6 of Speagle et al. (2014). These best-fit parameters have been adjusted for IMF, cosmology, SPS model, and emission line corrections. Column 4: $\log$ SFR predicted by each MS relation at $\log M_{*}=10$. Column 5: the median redshift. Column 6: redshift range. Column 7: stellar mass range. Column 8: survey data.

a The normalization was not adjusted by Speagle et al. (2014). We do not apply shifts to the stellar masses and SFRs as Grootes et al. (2013) make use of Chabrier (2003) IMF

b The normalization was not adjusted for systematics by Speagle et al. (2014). The MS trend of Cluver et al. (2017) is not shown in Figure 10 because KINGFISH galaxies were chosen to cover the full range of galaxy types, luminosities and mass properties, and local ISM environments rather than being a magnitude-limited sample.

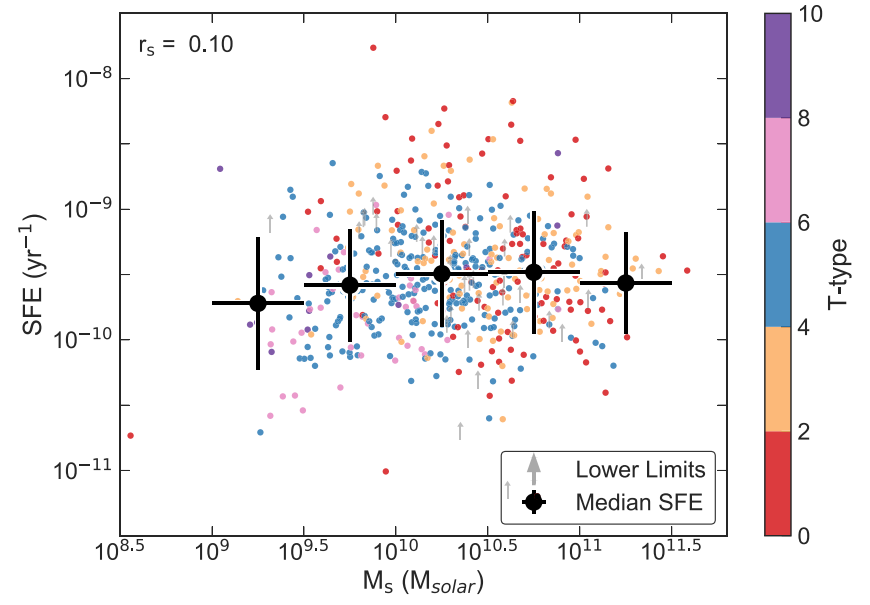

Figure 11. SFE and the stellar mass for the spiral sample. Median SFEs for each stellar mass bin are estimated including the $\mathrm{H}$ I mass upper limits for non$\mathrm{H}$ I detections, and the SFE medians and $1 \sigma$ values are listed in Table 7. For stellar masses ranging from $10^{9}$ to $10^{11.5} M_{\odot}$, the SFE for spirals remains constant at a median value of $\log \mathrm{SFE}=-9.57$ and $1 \sigma$ spread of 0.4 dex. SFE appears to be almost constant with T-type and stellar mass, despite the fact that high-mass galaxies with T-type $<2$ fall below the MS and may have commenced quenching.

Maddox et al. (2015) also measured the largest spin parameter to be $\lambda \sim 0.2$, confirming the upper limit on the halo spin parameter predicted by numerical $N$-body simulations of cold dark matter (Knebe \& Power 2008). They concluded that the upper limit on the H I fraction is set by the upper limit of the halo spin parameter due to the empirical correlation between the halo spin parameter and the H I fraction.

Obreschkow et al. (2016) found that for isolated local disk galaxies the fraction of atomic gas, $f_{\mathrm{atm}}$, is described by a stability model for flat exponential disks. To see if the observed upper limit to the H I fraction of the spiral sample can be explained by the upper limit of the halo spin parameter, we calculate the $f_{\text {atm }}$ relationship for $\lambda \approx 0.112$, following the method outlined in Obreschkow et al. (2016). Though $\lambda \sim 0.2$ is the maximum spin of a spherical halo, we choose to calculate the $f_{\text {atm }}$ at $\lambda \approx 0.112$ because $99 \%$ of galaxy halos are predicted to lie below $\lesssim 0.112$ (Bullock et al. 2001). We define
Table 7

The Median SFE and $1 \sigma$ Values of Each Stellar Mass Bin for the Spiral Sample Shown in Figure 11

\begin{tabular}{lccc}
\hline \hline $\log \left(M_{*}\right)$ & $\begin{array}{c}\text { Median } \log (\mathrm{SFE}) \\
\left(M_{\odot}\right)\end{array}$ & $\begin{array}{c}1 \sigma \\
(\mathrm{dex})\end{array}$ & $N$ \\
\hline 9.25 & -9.72 & 0.51 & 29 \\
9.75 & -9.58 & 0.43 & 128 \\
10.25 & -9.49 & 0.41 & 222 \\
10.75 & -9.48 & 0.47 & 164 \\
11.25 & -9.56 & 0.39 & 35 \\
\hline
\end{tabular}

the fraction of atomic gas as

$$
f_{\mathrm{atm}}=\frac{1.35 M_{\mathrm{HI}}}{M},
$$

where $M$ is the disk baryonic mass $\left(M=M_{*}+1.35 M_{\mathrm{HI}}\right)$ and the factor of 1.35 accounts for the universal helium fraction (Obreschkow et al. 2016). Obreschkow et al. (2016) models the rotation curve of spiral galaxies as

$$
f_{\text {atm }}=\min \left\{1,2.5 q^{1.12}\right\},
$$

where $q$ is the global stability parameter. This parameter is defined as:

$$
q=\frac{j \sigma}{G M}=0.22 \frac{\lambda}{0.03}\left(\frac{M}{10^{9} M_{\odot}}\right)^{-1 / 3},
$$

where $j$ is the baryonic specific angular momentum of the disk, $\sigma$ is the velocity dispersion of the atomic gas and mass is in units of $10^{9} M_{\odot}$. The global stability parameter is simplified by making two assumptions: first, that disk galaxies condense out of scale-free cold dark matter halos, and second, that $j \propto \lambda$ (Obreschkow \& Glazebrook 2014; Obreschkow et al. 2016). Under these assumptions, Equation (13) simplifies to:

$$
f_{\text {atm }}=\min \left\{1,0.5\left(\frac{\lambda}{0.03}\right)^{1.12}\left(\frac{M}{10^{9} M_{\odot}}\right)^{-0.37}\right\},
$$




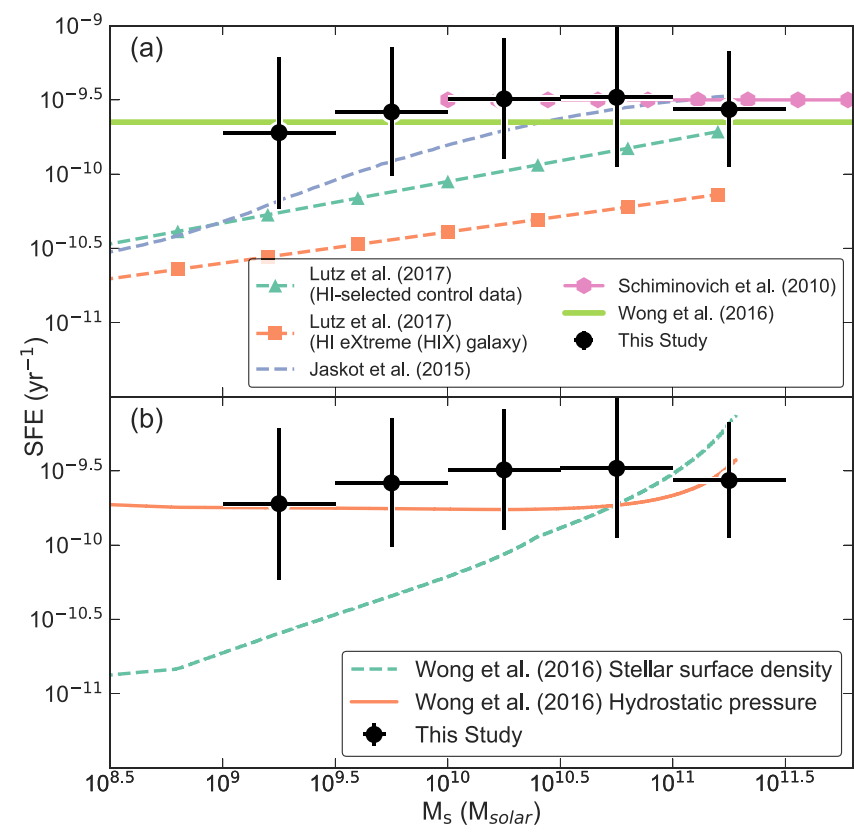

Figure 12. (Panel a) The median SFE and stellar mass for the spiral sample, alongside scaling relations observed in the prior literature (i.e., Schiminovich et al. 2010; Huang et al. 2012; Wong et al. 2016; Lutz et al. 2017) and (panel b) two models presented by Wong et al. (2016). H I-selected samples show that SFE increases with stellar mass, but this is an artifact of excluding low $\mathrm{H}$ I mass galaxies. Stellar mass-selected samples, on the other hand, show that SFE is constant with stellar mass, with normalization between $10^{-9.65}$ to $10^{-9.5} \mathrm{yr}^{-1}$. Of the two models presented by Wong et al. (2016), the hydrostatic pressure model provides the best agreement with our data.

and using Equation (12), this can be rearranged to give

$$
\begin{aligned}
\frac{M_{\mathrm{HI}}}{10^{9} M_{\odot}}= & \min \left\{\frac{M}{1.35 \times 10^{9} M_{\odot}},\right. \\
& \left.18 \lambda^{1.12}\left(\frac{M}{10^{9} M_{\odot}}\right)^{-0.63}\right\} .
\end{aligned}
$$

Figure 13 illustrates the comparison between Equation (16) and the empirical $\mathrm{H}$ I-stellar mass distribution of the spiral sample. We also include the predicted $f_{\text {atm }}$ curve for $\lambda \approx 0.03$, as this value of $\lambda$ corresponds to the mode of the empirically measured halo spin parameter distribution (Bullock et al. 2001). The median bins of the spiral sample are in agreement with this prediction of $f_{\mathrm{atm}}$, while the highest H I mass galaxies lie below the predicted upper limit for $f_{\text {atm }}$, when $\lambda=0.112$. We find that the model of Obreschkow et al. (2016) matches well with the empirical data of the spiral sample, consistent with the hypothesis that the upper limit of the $\mathrm{HI}$ fraction is set by that of the halo spin parameter.

\subsection{Why is SFE Constant?}

We find that SFE is constant across two orders of magnitude of stellar mass, which agrees with the findings of Catinella et al. (2010) and Wong et al. (2016), while disagreeing with others (e.g., Huang et al. 2012; Lutz et al. 2017). Wong et al. (2016) tested two models for molecular gas content within galaxies: one where molecular gas is a function of stellar surface density and another where it is a function of hydrostatic pressure. The stellar surface density prescription (Leroy et al. 2008; Zheng

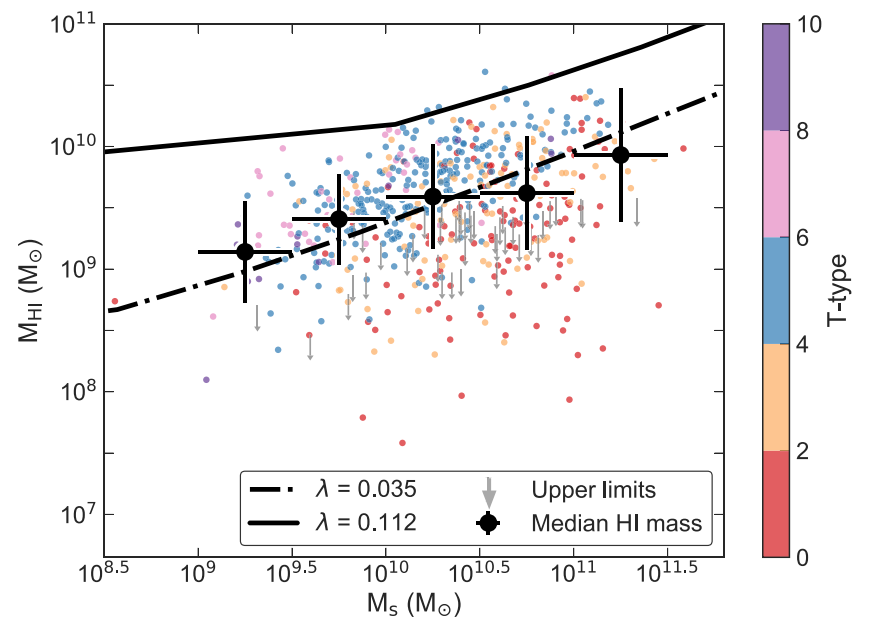

Figure 13. H I mass vs. stellar mass for the spiral sample. The solid and dashed black lines represent the model, Equation (16), for when $\lambda=0.112$ and 0.035 , respectively. The median bins of the spiral sample are in good agreement with the model of Obreschkow et al. (2016), as they lie on the expected mean $f_{\mathrm{atm}}$ (for $\lambda=0.035$ ). The upper $\mathrm{HI}$ masses of the spiral sample line up with the expected $f_{\text {atm }}$ for $\lambda=0.112$, and therefore the maximum $\mathrm{H}$ I mass for a given stellar mass is determined by the upper limit in the halo spin parameter.

et al. 2013) defines the molecular-to-atomic ratio, $R_{\mathrm{mol}}$, as

$$
R_{\mathrm{mol}, s}=\frac{\Sigma_{*}}{81 M_{\odot} \mathrm{pc}^{-1}}
$$

where $\Sigma_{*}$ is the stellar surface density. The $R_{\mathrm{mol}}$ for the hydrostatic pressure prescription (Zheng et al. 2013) is defined as

$$
R_{\mathrm{mol}, p}=\left(\frac{P_{h}}{1.7 \times 10^{4} \mathrm{~cm}^{-3} \mathrm{~K} k_{\mathrm{B}}}\right)^{0.8},
$$

where $P_{h}$ is the hydrostatic pressure (Elmegreen 1989) and $k_{\mathrm{B}}$ is the Boltzmann constant.

Similarly to Wong et al. (2016), we find that the constant SFE can be described by a model of the marginally stable disk, while the hydrostatic pressure model provides a better prescription for estimating the SFE and molecular-to-atomic ratio. For massive galaxies with large optical disks, previous studies (e.g., Leroy et al. 2008; Wong et al. 2013) observed a correlation between $R_{\mathrm{mol}}$ and stellar surface density. The two models given by Equations (17) and (18) also predict similar $R_{\text {mol }}$ and integrated SFE for high-mass galaxies (Wong et al. 2016). But for low mass galaxies, the stellar surface density prescription does not predict the observed SFE because this prescription is unable to convert the $\mathrm{HI}$ to molecular hydrogen, and underestimates the amount of molecular hydrogen in regions with low stellar surface densities. Therefore, this method underestimates the SFR and SFE in dwarf galaxies. While the stellar surface density model predicts that SFE will decrease for smaller stellar mass galaxies, the hydrostatic pressure model predicts a higher molecular hydrogen content for low mass galaxies, and therefore a constant SFE with stellar mass, agreeing with the empirical data.

We note that Obreschkow et al. (2016) predict that most of the baryons in dwarf galaxies are in the form of $\mathrm{HI}$ gas $\left(f_{\text {atm }}=1\right)$ and therefore have low SFE because these systems are inefficient at converting their $\mathrm{HI}$ gas to molecular gas. We do not observe a decrease in SFE because these galaxies are 
below the stellar mass range probed in our spiral sample. Our lowest stellar mass bin of $10^{9.0}$ and $10^{9.5} M_{\odot}$ hints at a turnover for the low mass dwarf, as predicted by Obreschkow et al. (2016). Combining the next generation of H I survey, WALLABY, with $20 \mathrm{~cm}$ radio continuum from ASKAP, we will measure the H I properties and SFR of $\sim 600,000$ galaxies, including a large number of dwarf galaxies populating the low mass end of Figure 12. Observing these low stellar mass dwarfs will provide a more complete picture about $\mathrm{H}$ I content and how efficiently dwarfs convert $\mathrm{H}$ I to molecular gas.

\section{Summary}

We have measured the relationship between H I mass, stellar mass, and SFE by using HICAT, archival H I data, and new WISE photometry. For this work, we provide new WISE aperture photometry for 3831 out of 4315 sources of HICAT and created three samples, an $\mathrm{H}$ I-selected sample, a spiral sample, and an $M_{s}$ sample. We find the following:

1. Sample selection and biases are critical when interpreting and comparing measured relationships between $\mathrm{H}$ I mass, stellar mass, and SFE. H I-selected samples often exclude H I-poor galaxies (unlike stellar mass-selected samples) resulting in measurements of high median $\mathrm{H}$ I masses and low median SFEs.

2. H I mass increases with stellar mass for the spiral sample with a power-law index of 0.34 . Also, at a given stellar mass, H I mass increases with T-type and dispersion in $\mathrm{H}$ I masses narrows for individual T-types. For example, for the $10^{10} M_{\odot}$ to $10^{10.5} M_{\odot}$ stellar mass bin, the median H I mass and scatter is $10^{9.26} M_{\odot}$ and 0.59 dex for T-types 0 to 2 and the $10^{9.72} M_{\odot}$ and 0.31 dex for T-types 6 to 8 .

3. H I mass is constant with stellar mass for the $M_{s}$ sample. While HI mass increases with stellar mass for spiral galaxies, the fraction of elliptical galaxies with little $\mathrm{H} \mathrm{I}$ gas also increases with stellar mass, producing the observed flat relation.

4. The observed upper limit to the $\mathrm{H}$ I-stellar mass distribution of the spiral sample is consistent with the predicted H I-stellar mass curve for the upper limit for the halo spin parameter $(\lambda=0.112)$. This is consistent with the hypothesis that the maximum $\mathrm{H}$ I fraction is set by that of the halo spin parameter.

5. For a subsample of the spiral sample with stellar mass $\leqslant 10^{10.5} M_{\odot}$, we measure the MS to be $\log$ SFR $=0.7$ $\left(\log M_{*}-10\right)-0.09$ with a $1 \sigma$ scatter of 0.27 dex. We see evidence of mass quenching (e.g., Kauffmann et al. 2003) as the median SFR is constant for spiral galaxies with stellar masses $>10^{10} M_{\odot}$.

6. For the spiral sample, SFE is constant $\left(=10^{-9.57} \mathrm{yr}^{-1} \pm\right.$ 0.44 dex) for 2.5 orders of magnitude in stellar mass and agrees with comparable measurements of stellar massselected samples of galaxies (Catinella et al. 2010; Schiminovich et al. 2010). This result is in broad agreement with the hydrostatic pressure model (Wong et al. 2016).

7. SFE is constant as a function of T-type and is constant for spiral galaxies that show evidence of mass quenching.

We would like to thank M. Cluver, T. Dolley, A. Groszek, V. Kilborn, K. Lutz, N. Maddox, G. Meurer, D. Obreschkow, and I. Wong for the useful and insightful discussions.

This publication makes use of data products from the Widefield Infrared Survey Explorer, which is a joint project of the University of California, Los Angeles, and the Jet Propulsion Laboratory/California Institute of Technology, funded by the National Aeronautics and Space Administration. This research has made use of the NASA/IPAC Extragalactic Database (NED) which is operated by the Jet Propulsion Laboratory, California Institute of Technology, under contract with the National Aeronautics and Space Administration and the HyperLeda database (http://leda.univ-lyon1.fr).

\section{Appendix}

Table 8 provides a full list of parameters of HICAT + WISE (H I-WISE) catalog that is available in machine-readable format. 
Table 8

H I-WISE Parameter Descriptions

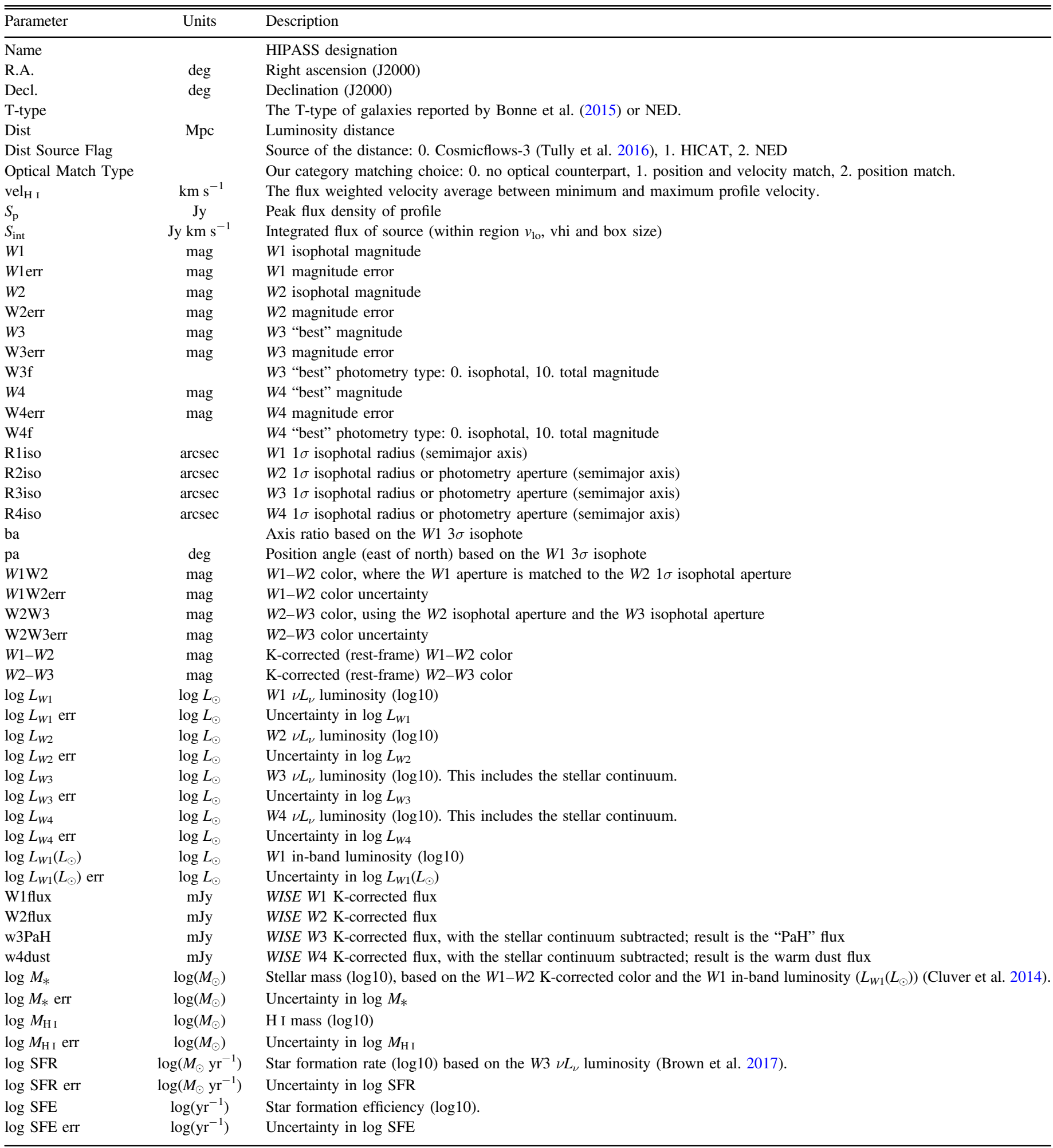

(This table is available in its entirety in machine-readable form.) 


\section{ORCID iDs}

Vaishali Parkash (1) https://orcid.org/0000-0003-3982-6304

Michael J. I. Brown (1) https://orcid.org/0000-0002-1207-9137

T. H. Jarrett (i) https://orcid.org/0000-0002-4939-734X

Nicolas J. Bonne 구 https://orcid.org/0000-0001-9569-8808

\section{References}

Barnes, D. G., Staveley-Smith, L., de Blok, W. J. G., et al. 2001, MNRAS, 322, 486

Boissier, S., \& Prantzos, N. 2000, MNRAS, 312, 398

Bolatto, A. D., Wolfire, M., \& Leroy, A. K. 2013, ARA\&A, 51, 207

Bonne, N. J., Brown, M. J. I., Jones, H., \& Pimbblet, K. A. 2015, ApJ, 799,160

Brinchmann, J., Charlot, S., White, S. D. M., et al. 2004, MNRAS, 351, 1151 Brown, M. J. I., Moustakas, J., Kennicutt, R. C., et al. 2017, ApJ, 847, 136 Bullock, J. S., Kolatt, T. S., Sigad, Y., et al. 2001, MNRAS, 321, 559

Calzetti, D., Kennicutt, R. C., Engelbracht, C. W., et al. 2007, ApJ, 666, 870 Catinella, B., Schiminovich, D., Cortese, L., et al. 2013, MNRAS, 436, 34

Catinella, B., Schiminovich, D., Kauffmann, G., et al. 2010, MNRAS, 403, 683 Catinella, B., Schiminovich, D., Kauffmann, G., et al. 2012, A\&A, 544, A65 Chabrier, G. 2003, PASP, 115, 763

Chen, Y.-M., Wild, V., Kauffmann, G., et al. 2009, MNRAS, 393, 406

Cluver, M. E., Jarrett, T. H., Dale, D. A., et al. 2017, ApJ, 850, 68

Cluver, M. E., Jarrett, T. H., Hopkins, A. M., et al. 2014, ApJ, 782, 90

Cutri, R. M., Wright, E. L., Conrow, T., et al. 2013a, Explanatory Supplement to the WISE All-Sky Data Release Products, Tech. Rep. 1

Cutri, R. M., Wright, E. L., Conrow, T., et al. 2013b, yCat, 2328

Doyle, M. T., \& Drinkwater, M. J. 2006, MNRAS, 372, 977

Doyle, M. T., Drinkwater, M. J., Rohde, D. J., et al. 2005, MNRAS, 361, 34

Driver, S. P., Norberg, P., Baldry, I. K., et al. 2009, A\&G, 50, 5.12

Elbaz, D., Daddi, E., Le Borgne, D., et al. 2007, A\&A, 468, 33

Elmegreen, B. G. 1989, ApJ, 338, 178

Fixsen, D. J., Cheng, E. S., Gales, J. M., et al. 1996, ApJ, 473, 576

Gabor, J. M., Davé, R., Finlator, K., \& Oppenheimer, B. D. 2010, MNRAS, 407, 749

Grootes, M. W., Tuffs, R. J., Popescu, C. C., et al. 2013, ApJ, 766, 59

Helou, G., Roussel, H., Appleton, P., et al. 2004, ApJS, 154, 253

Huang, S., Haynes, M. P., Giovanelli, R., \& Brinchmann, J. 2012, ApJ, 756, 113

Huang, T.-C., Goto, T., Hashimoto, T., Oi, N., \& Matsuhara, H. 2017, arXiv: 1712.01861

Huchtmeier, W. K., \& Richter, O.-G. 1989, A General Catalog of HI Observations of Galaxies: The Reference Catalog (Berlin: Springer)

Jarrett, T. H., Chester, T., Cutri, R., et al. 2000, AJ, 119, 2498

Jarrett, T. H., Cluver, M. E., Magoulas, C., et al. 2017, ApJ, 836, 182

Jarrett, T. H., Cohen, M., Masci, F., et al. 2011, ApJ, 735, 112

Jarrett, T. H., Masci, F., Tsai, C. W., et al. 2012, AJ, 144, 68

Jarrett, T. H., Masci, F., Tsai, C. W., et al. 2013, AJ, 145, 6

Jaskot, A. E., Oey, M. S., Salzer, J. J., et al. 2015, ApJ, 808, 66

Jones, D. H., Read, M. A., Saunders, W., et al. 2009, MNRAS, 399, 683
Kauffmann, G., Heckman, T. M., Tremonti, C., et al. 2003, MNRAS, 346, 1055

Kennicutt, R. C., Jr. 1998, ARA\&A, 36, 189

Kereš, D., Katz, N., Fardal, M., Davé, R., \& Weinberg, D. H. 2009, MNRAS, 395,160

Knebe, A., \& Power, C. 2008, ApJ, 678, 621

Leroy, A. K., Walter, F., Brinks, E., et al. 2008, AJ, 136, 2782

Lintott, C., Schawinski, K., Bamford, S., et al. 2011, MNRAS, 410, 166

Lintott, C. J., Schawinski, K., Slosar, A., et al. 2008, MNRAS, 389, 1179

Lutz, K. A., Kilborn, V. A., Catinella, B., et al. 2017, MNRAS, 467, 1083

Maddox, N., Hess, K. M., Obreschkow, D., Jarvis, M. J., \& Blyth, S.-L. 2015, MNRAS, 447, 1610

Mainzer, A., Bauer, J., Cutri, R. M., et al. 2014, ApJ, 792, 30

Makarov, D., Prugniel, P., Terekhova, N., Courtois, H., \& Vauglin, I. 2014, A\&A, 570, A13

Masters, K. L., Crook, A., Hong, T., et al. 2014, MNRAS, 443, 1044

Meidt, S. E., Schinnerer, E., Knapen, J. H., et al. 2012, ApJ, 744, 17

Meidt, S. E., Schinnerer, E., van de Ven, G., et al. 2014, ApJ, 788, 144

Meyer, M. J., Zwaan, M. A., Webster, R. L., et al. 2004, MNRAS, 350, 1195

Mirabel, I. F., \& Sanders, D. B. 1988, ApJ, 335, 104

Noeske, K. G., Weiner, B. J., Faber, S. M., et al. 2007, ApJL, 660, L43

Obreschkow, D., \& Glazebrook, K. 2014, ApJ, 784, 26

Obreschkow, D., Glazebrook, K., Kilborn, V., \& Lutz, K. 2016, ApJL, 824, L26

Oliver, S., Frost, M., Farrah, D., et al. 2010, MNRAS, 405, 2279

Paturel, G., Petit, C., Prugniel, P., et al. 2003, A\&A, 412, 45

Ponomareva, A. A., Verheijen, M. A. W., Papastergis, E., Bosma, A., \& Peletier, R. F. 2018, MNRAS, 474, 4366

Prochaska, J. X., \& Wolfe, A. M. 2009, ApJ, 696, 1543

Rodighiero, G., Daddi, E., Baronchelli, I., et al. 2011, ApJL, 739, L40

Salim, S., Rich, R. M., Charlot, S., et al. 2007, ApJS, 173, 267

Schiminovich, D., Catinella, B., Kauffmann, G., et al. 2010, MNRAS, 408, 919

Schruba, A., Leroy, A. K., Walter, F., et al. 2011, AJ, 142, 37

Serra, P., Oosterloo, T., Morganti, R., et al. 2012, MNRAS, 422, 1835

Speagle, J. S., Steinhardt, C. L., Capak, P. L., \& Silverman, J. D. 2014, ApJS, 214, 15

Springob, C. M., Haynes, M. P., Giovanelli, R., \& Kent, B. R. 2005, ApJS, 160,149

Stern, D., Assef, R. J., Benford, D. J., et al. 2012, ApJ, 753, 30

Taylor, E. N., Hopkins, A. M., Baldry, I. K., et al. 2011, MNRAS, 418, 1587

Tully, R. B., Courtois, H. M., \& Sorce, J. G. 2016, AJ, 152, 50

Véron-Cetty, M.-P., \& Véron, P. 2010, A\&A, 518, A10

Wong, O. I., Meurer, G. R., Zheng, Z., et al. 2016, MNRAS, 460, 1106

Wong, T., Xue, R., Bolatto, A. D., et al. 2013, ApJL, 777, L4

Wright, E. L., Eisenhardt, P. R. M., Mainzer, A. K., et al. 2010, AJ, 140, 1868

Wuyts, S., Förster Schreiber, N. M., Lutz, D., et al. 2011, ApJ, 738, 106

York, D. G., Adelman, J., Anderson, J. E., et al. 2000, AJ, 120, 1579

Zahid, H. J., Dima, G. I., Kewley, L. J., Erb, D. K., \& Davé, R. 2012, ApJ, 757,54

Zheng, Z., Meurer, G. R., Heckman, T. M., Thilker, D. A., \& Zwaan, M. A. 2013, MNRAS, 434, 3389

Zwaan, M. A., Meyer, M. J., Webster, R. L., et al. 2004, MNRAS, 350, 1210 